
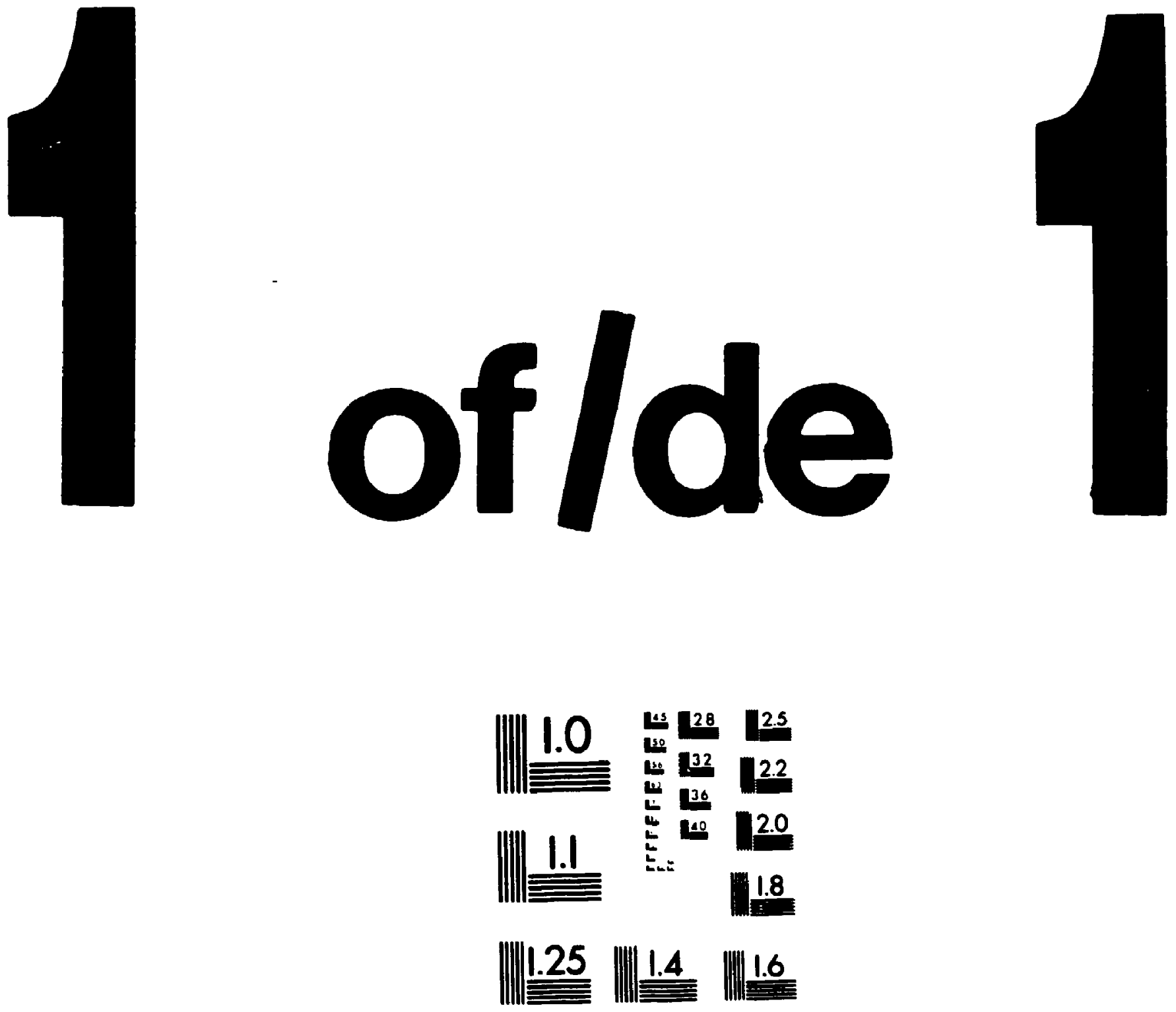

MICAOCOPY RESOLUTION TEST CHART

NATIONAL BUREAU OF STANDARDS

STANOARO REFERENCE MATERIAL 10100

(ANSI and ISO TEST CHART NO 2) 
Acquisitions and

Bibliographic Services Branch

395 Wellungton Street

Ottawa, Ontano

KIA ON4
Direction des acquisitions et des senvices bibliographrques

395. ne Wrilington

Ontawa $(0-\tan )$

KIA ONA
NOTICE

The quality of this microform is heavily dependent upon the quality of the original thesis submitted for microfilming. Every effort has been made to ensure the highest quality of reproduction possible.

If pages are missing, contact the university which granted the degree.

Some pages may have indistinct print especially if the original pages were typed with a poor typewriter ribbon or if the university sent us an inferior photocopy.

Reproduction in full or in part of this microform is governed by the Canadian Copyright Act, R.S.C. 1970, C. C-30, and subsequent amendments.
La qualité de cette microforme dépend grandement de la qualité de la thèse soumise au microfilmage. Nous avons tout fait pour assurer une qualité supérieure de reproduction.

S'il manque des pages, veuillez communiquer avec l'université qui a conféré le grade.

La qualité d'impression de certaines pages peut laisser à désirer, surtout si les pages originales ont été dactylographiées à l'aide d'un ruban usé ou si l'université nous a fait parvenir une photocopie de qualité inférieure.

La reproduction, même partielle, de cette microforme est soumise à la Loi canadienne sur le droit d'auteur, SRC 1970, c. C-30, et ses amendements subséquents. 


\title{
Bounds on the Interpolation Capacity of Feed-Forward Neural Networks
}

\author{
by \\ Scott Reynolds \\ A thesis submitted to \\ the Faculty of Graduate Studies and Research \\ in partial fulfillment of \\ the requirements for the degree of \\ Master of Computer Science \\ Ottawa-Carleton Institute for Computer Science \\ School of Cumputer Science
}
Carleton University
Ottawa, Ontario
August 12, 1993

(c) Copyright

1993, Scott Reynolds 
National Library

of Canada

Acquisitions and

Bibliographic Services Branch

395 Wollington Streat

Ottawa. Ontario

KIA ONA
Bibliotheque nationale

du Canada

Direction des acm visitions ef

des services bil... phiques

395, ne Welington

Ottewa (Ontario)

KIAONA

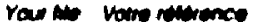

Ou whe Notre rewnerce
The author has granted an irrevocable non-exclusive licence allowing the National Library of Canada to reproduce, loan, distribute or sell copies of his/her thesis by any means and in any form or format, making this thesis available to interested persons.
L'auteur a accordé une licence irrévocable et non exclusivé permettant à la Bibliothéque nationale du Canada de reproduire, prêter, distribuer ou vendre des copies de sa thèse de quelque manière et sous quelque forme que ce soit pour mettre des exemplaires de cette these a la disposition des personnes intéressées.

L'auteur conserve la propriété du droit d'auteur qui protège sa these. Ni la these ni des extraits substantiels de celle-ci ne doivent être imprimés ou autrement reproduits sans son sutorisation.

ISBN $\quad 0-315-89817-8$ 
Name Sc. Tt Reyned d. S

Dissertation Abstracts Intornotional is arranged by broad, generol subject categories. Pleose select the one subject which most nearty describes the content of your dissertation. Enter the corresponding four-digit code in the spaces provided.

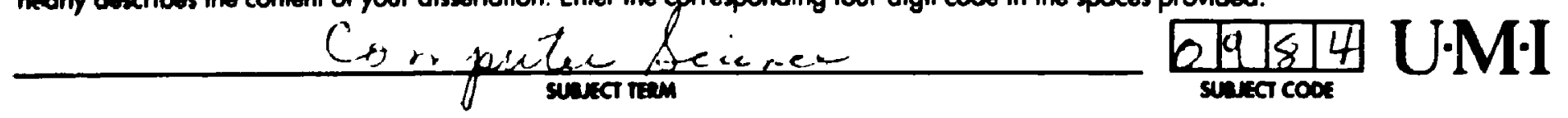

\section{Subjinct Cokpories}

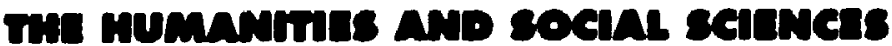

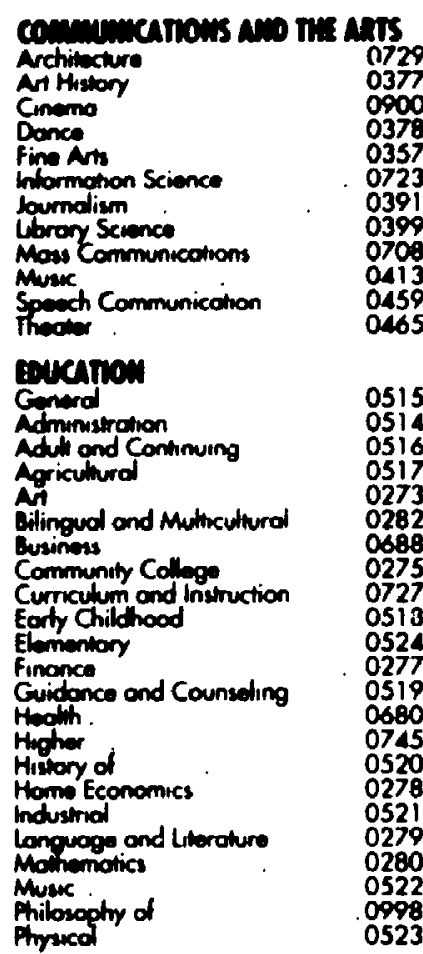

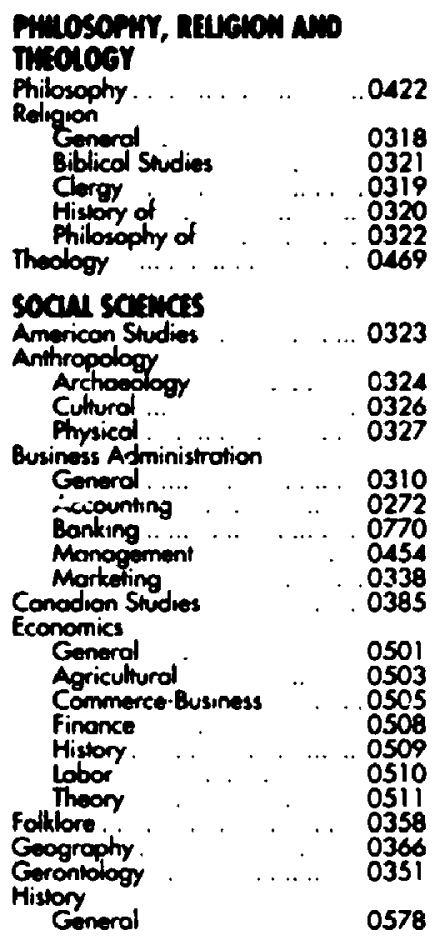

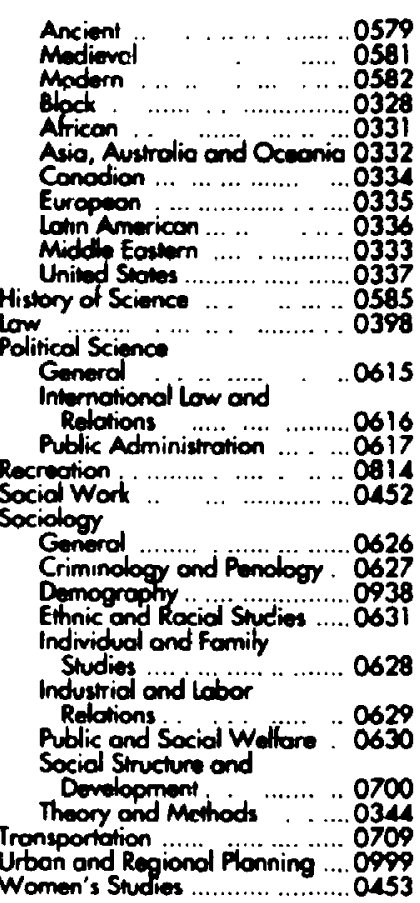

Wran and Regionol Flanning .....0\%9

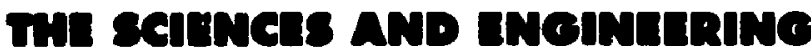

\section{mosicu satios}

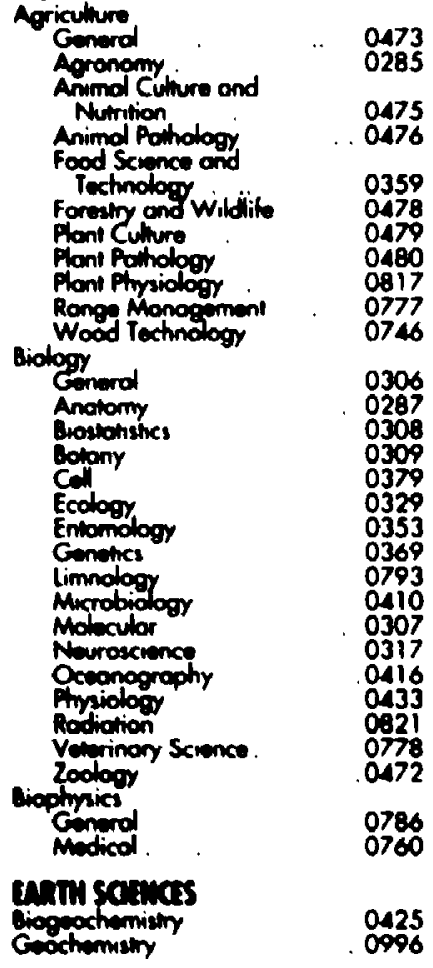

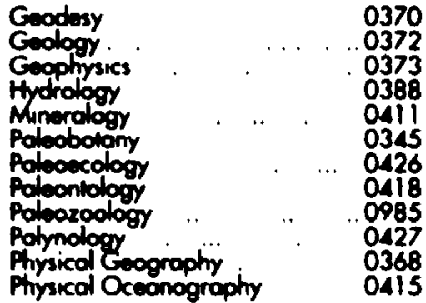

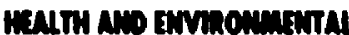

sainas

Envronmentol Scrences $\quad .0768$

Hoolth Sciences

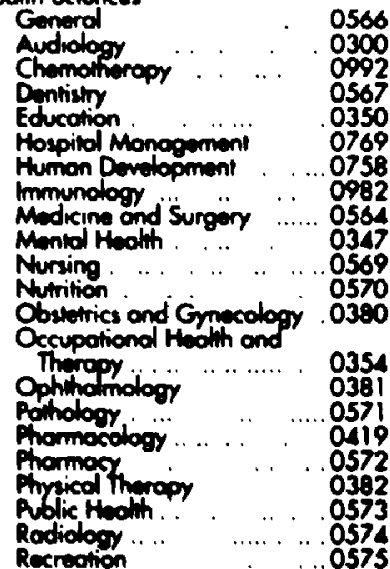

Speoch Pathology. $\quad 0460$

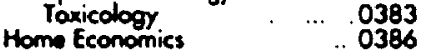

\section{PHrskal satancts}

Pure Sciences

Chemistivy

Genorol ... 0485

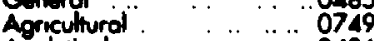

Analytical $\quad . . .0486$

Bioctiemisiny . ........ 0487

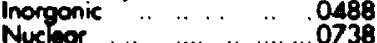

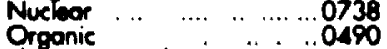

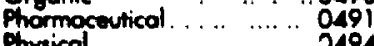

Physical ...

Roymer ... …......... 045

Mothematics ............... 0405

Phrsics

Generol .................... 0605

Acoustics... . . . 0986

Astronomy and

Astrophyies ............. 0606

Almospheric Science ..........0608

$\begin{array}{ll}\text { Atomic } & 0746 \\ \text { Electronics ond Electricity } .0607\end{array}$

Electronics and Eloctricity

Hioh Enery............0798

Fhid and Plasmo ... . 0759

Nucteor ................................ .0615

Optics ............................... 0752

0756

Solid Stote .................... . OS1

Stotistics ................................ 0463

Appind Scinces

Applind Muchonica .................0346

Computer Science .................... 0984
Engunoering

$\begin{array}{lllll}\text { Generd } & . & \ldots & \ldots & .0537\end{array}$

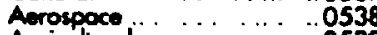

Agricutural ................ 0539

Automotive …................0540

Chemicol ........... . ......0542

Civil ....................... 0543

Electronics and Electricol .......0544

thot and Thermodynomics .. 0348

Hydrautic. .................... 0545

Industriol ..................... 0546

Moterials Science . . ... . . ...079

Mechonicol. ..............0548

Motollurgy ........................ 0743

Mining . ....................055

Nucteor. .. .................. 055

Pockoging ................... 0549

Sonitary and Municipol .... 55

System Science ... . ........... $07 \%$

Geowehnology ......................... 0428

Operotions Revoorch .................. 0796

Plastics Technology ....................0795

psrawores

Generol ......................0821

Clinicat ............................... 0622

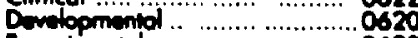

Experimentol ..

Industriod ....

Personolity........................ \$825

Poychobiclooy. ................. 0349

Pychomentice ......................... 063

Sociol .................................. Q451 
The undersigned hereby recommend to

The Faculty of Graduate Studies and Rescarch acceptance of the thesis.

Bounds on the Interpolation Capacity of Feed-Forward Neural Networks submitted by

Scott Reynolds

in partial fulfilment of the requirenents

for the degree of Master of Computer Sicienco

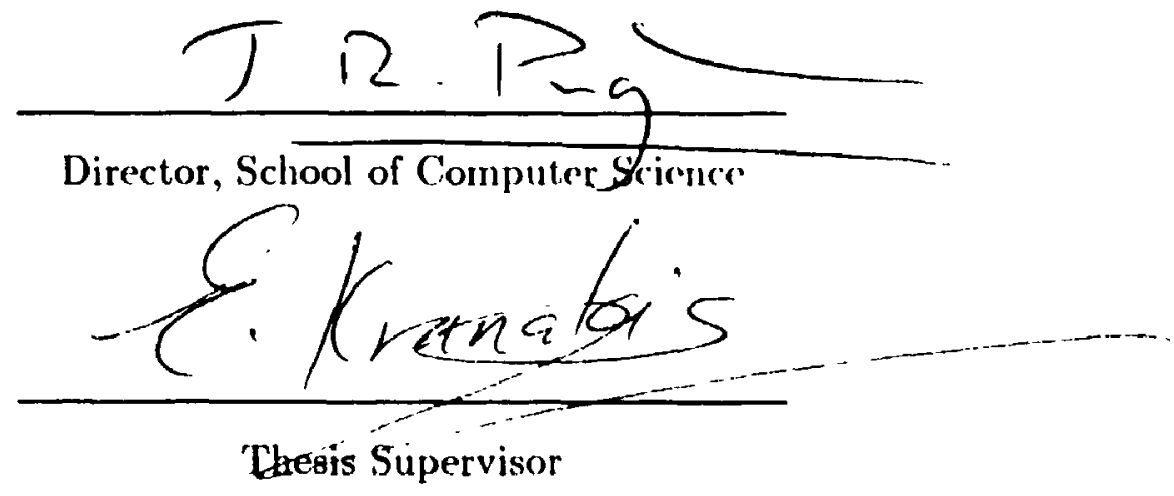

Carleton University

August 121993 


\section{Abstract}

The interpolation capacity of a neural network $N$ is the smallest number $i$ such that all sets of lata of size $i$, or less, can be realized by the network $N$. The networks considered here are analog, they compute functions from $\Re \rightarrow \Re$. However, the results here also apply to networks that compute functions from $\Re^{n} \rightarrow \Re$. Results have been found for the interpolation capacity of various classes of networks. For a one layer network having $k$ nodes with the standard sigmoid as the activation function, the previous known upper bound is improved to $2 k$. For a two layer network having $k$ nodes on the first layer and $d$ nodes on the second layer with the standard sigmoid as the activation function, the lower bound is determined to be $k d$. For a two layer network having $k$ nodes on the first layer and $d$ nodes on the second layer with the piecewise linear $\pi$ function as the activation function, the lower bound is determined to be $k c$. 


\section{Acknowledgments}

I thank my supervisor, Evangelos Kranakis, for his enthusiasm. for listening to IIIy ideas and for letting me do what I wanted to do.

I thank John Pugh for allowing me the great pleasure of instructing undergraduate courses. 


\section{Contents}

Abstract

Acknowledgnents $\quad$ iv

List Of Figures $\quad$ vi

1 Introduction 1

2 Related Research 10

3 An Upper Bound $\quad 15$

$4 \pi$ Bounds $\quad 18$

5 Asymptotic Sigmoid Bounds $\quad 32$

$\begin{array}{llr}6 & \text { Conclusion } & 47\end{array}$

$\begin{array}{ll}\text { Bibliography } & 49\end{array}$ 


\section{List of Figures}

1.1 One layer network. one input $\ldots \ldots \ldots \ldots$

1.2 One laver network, multiple inputs $\ldots \ldots \ldots \ldots$

1.3 Two layer network, one input $\ldots \ldots \ldots \ldots \ldots$

1.4 Neural network node . . . . . . . . . . . . . . . . . 6

1.5 Standard sigmoid function $\ldots \ldots \ldots \ldots \ldots$

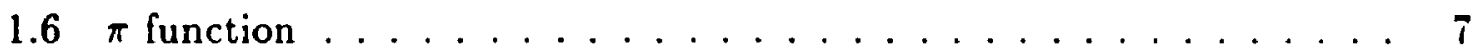

1.7 Heaviside step function $\ldots \ldots \ldots \ldots \ldots \ldots$

3.1 The standard sigmoid and its derivative $\ldots \ldots \ldots \ldots$

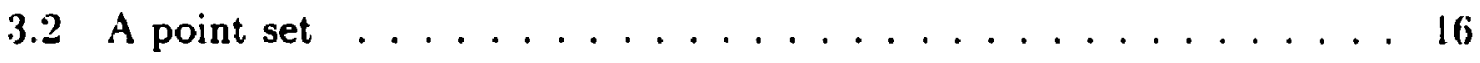

4.1 Weight assignments of the network . . . . . . . . . . 19

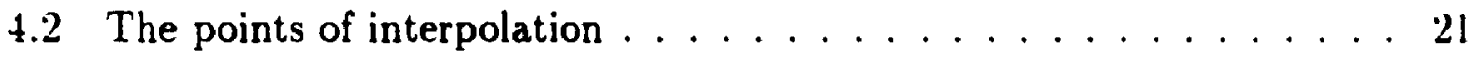

5.1 Outputs of a $2-2$ network $\ldots \ldots \ldots \ldots \ldots$ 


\section{Chapter 1}

\section{Introduction}

Neural Networks are a model of computation that are inspired by neurons found in the brain. One of the properties of the brain is that it is able to learn and adapt. Neural Networks also have this property and various training algorithms have been developed that make them learn some data or items of information. Though they have yet to achieve anything like the simulation of human consciousness, remarkable results have been obtained with them. The best computer program in the world for the gane of backgammon is a neural network $[3,28,29]$. It has been trained to play at such a high level that it is nearly as good as the very best human players in the world. Another interesting result is that a neural network that initially does not know any strategy about backgammon has achieved master-level play by playing against itself and learning from the results.

'The current most common form of neural network training is that the data it must learn is repeatedly presented to it until it knows the correct answer for all the items in the data set. It makes small changes to its structure each time an item from the data set is presented and after enough time the network should be able to give the correct response for all the items in the data set. This is the philosophy behind the best known training method, the back-propagation algorithm [24].

There are problems with training neural networks. One of the most important 
ones is that given a neural network of a certain size it may be impossible for it ever to learn the entire data set exactly. It has been given 100 much data to learn for its size. As an analogy, a polynomial of a fixed degree may not be able to interpolate a set of points. but a polynomial of greater degree will be able to. The size of the data set for which it is possible for a neural network to aluras be trained is called the interpolation capacity of the network. This is the subject of this thesis.

Mathematically, neural networks are circuits that compute functions. They also offer an exciting model for analog computation. 'That is, they can compute functions from $\Re^{n}$ to $\Re$. In this way they differ from traditional branches of circuit theory that. study the computation of boolean functions from $\{0,1\}^{n}$ to $\{0,1\}$. Two common classes of circuits are those with and, or, and not gates and those with threshold gates.

A feed-forwaid neural network is an acyclic neural network. 'That is, it. does not have feed-backward $t$ dges. If cycles are allowed they are called recurrent neural networks. Both types are currently in use. However, they have complet aly different properties and are used in different ways. Feed-forward networks start their computation from an input that feeds forward the input to all the nodes on ihe first layer. Each of the nodes on the first layer does a calculation on the input and feeds its result forward to the next layer, and so on. Recurrent networks operate in a similar manner, but the nodes on a layer can feed to a layer below, whereas a feed forward network can never feed backwards. Since a recurrent network has feed-backward edges, the computation keeps cycling until, hopefully, a steady final output is obtained. It may be the case that there is not a steady final output. Feed-forward networks do not have this problem and the time it takes to do a computation is dependent only on the depth of the network. This thesis is concerned only with feed-forward neural networks.

Typically, in a feed forward nenral network the computation nodes are arranged in layers. The terminology is not standard and sometimes the output node is counted as a layer. In this thesis, a $d$-layer network has $d$ layers of computation nodes. 'The final sum of the last layer that gives the output is not counted here. Figure 1.1 


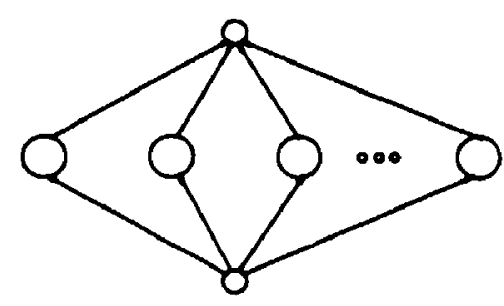

Figure 1.1: One layer network, one input

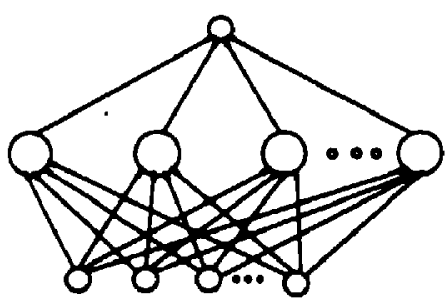

Figure 1.2: One layer network, multiple inputs

shows a one-layer network with one input. The input is at the bottom. Then there are some computation nodes and at the top is the output. A neural network with one real input and appropriate computation nodes, that evaluate to real values, calculates a function $\Re \rightarrow \Re$. Figure 1.2 shows a one-layer network with multiple inputs. All the inputs are fed to each computation node. With $n$ real inputs and appropriate computation nodes, it calculates a function $\Re^{n} \rightarrow \Re$. Figure 1.3 depicts a two-layer network with one input. All the outputs from the first layer are used as inputs to the second layer. Such a network would calculate a function $\Re \rightarrow \Re$. One would expect that the network in figure 1.3 has more interpolational power than the one in figure 1.1, if they had the same number of nodes on each layer. This is indeed the case for a wide range of computational nodes and is one of the main results of this thesis.

The romputations that the individual nodes of a network do are very simple. First a weighted sum of the inputs is calculated. Then only the amount over a certain threshold is passed to an activation function. See figure 1.4. If a node has $k$ inputs with variables $X_{1}, X_{1}, \ldots, X_{k}$ and activation function $\theta$ then the computation 


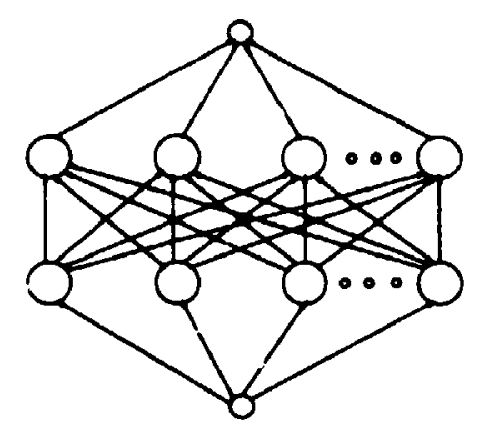

Figure 1.3: Two layer network, one input.

performed by the node would be $\theta\left(W_{1} X_{1}+W_{2} X_{2}+W_{3} X_{3}+\cdots+W_{k} \cdot X_{k}-T\right)$. 'The $W^{\cdot " s}$ represent the weights of the input edges to the node and $T$ the threshold of the node. All neural network training algorithms must determi:ze the correct assignments of the weights and thresholds in order for the network to interpolate the data corrertly.

Neural Networks are used with different types of activation functions. However, once an activation function is chosen it is usually used for all the nodes in the network. The most important class of activation functions are the sigmoids. $\Lambda$ sigmuid is a function, $\Re \rightarrow \Re$, whose domain have, or asymptotically approach, a constant value, as the range goes to positive infinity, and whose domain have, or asymptotically approach, a different constant value, as negative infinity is approached. 'The' most common sigmoid in use is called the standard sigmoid, denoted by $\theta_{s}$, and is given by

$$
\theta_{s}(x)=\frac{1}{1+e^{-x}}
$$

See figure 1.5. It is continuous, infinitely smooth and symmetric around zero. When $x \rightarrow \infty$ the function asymptotically approaches one and when $x \rightarrow-\infty$ the function asymptotically approaches zero.

Formally, we have the following definitiois.

Definition A sigmoid $\theta$ is an asymptotic sigmoid if the following two conditions hold:

1. $\lim _{x \rightarrow \infty} \theta(x)=C_{1}$ and $\lim _{x \rightarrow-x} \theta(x)=C_{2}$, with $C_{1} \neq C_{2}^{\prime}$. 
2. $\forall x \exists y($ surch that $\theta(s)<\theta(y)$ and $\theta(x)>\theta(y))$.

Two mon-asymptotic sigmoids are the $\pi$ and $\mathcal{H}$ functions. The $\pi$ function is defined is

$$
\pi(x)= \begin{cases}0 & \text { if } x \leq 0 \\ 1 & \text { if } x \geq 1 \\ x & \text { otherwise }\end{cases}
$$

See figure 1.6. It is sometimes called the pierewise linear sigmoid because it consists of three linear segments. In a sense, this is the simplest analog sigmoid. It has the same limiting values as the standard sigmoid. It is continuous, but not smooth. Since it is not smooth it is not used much experimentally, however it is of theoretical interest bec ause of its simplicity.

The last common sigmoid is the Heaviside step function. Defined by

$$
\mathcal{H}(x)= \begin{cases}0 & \text { if } x \leq 0 \\ 1 & \text { if } x>0\end{cases}
$$

See figure 1.7. Once again it has the same limiting values as the $\theta_{s}$ and $\pi$ functions. However, it is neither continuous nor smooth at zero. Also it outputs only boolean values. When neural networks with the Heaviside step function are used for the activation functions and the network is only assumed to have boolean inputs the network is ralled a threshold circuit. These have been much studied since the 1960's by traditional circuit theorists. If only two inputs to a node are allowed and only weights of one are used then the resulting network is the traditional circuit cornposed of and and or gates. If weights of negative one and zero are also allowed then the circuit can have no' gates.

Activation functions other than sigmoids are used, such as, radial basis functions [16, 23,26], and piecewise polynomials [30]. This thesis is concerned only with sigmoids which output real values, that is analog computation. Networks with the Heaviside function only have boolean outputs and have been much studied in thia past and already a lot is known about them. However, the study of analog compu. tation with real valued sigmoids is just starting and this thesis makes an important contribution to this new field. 


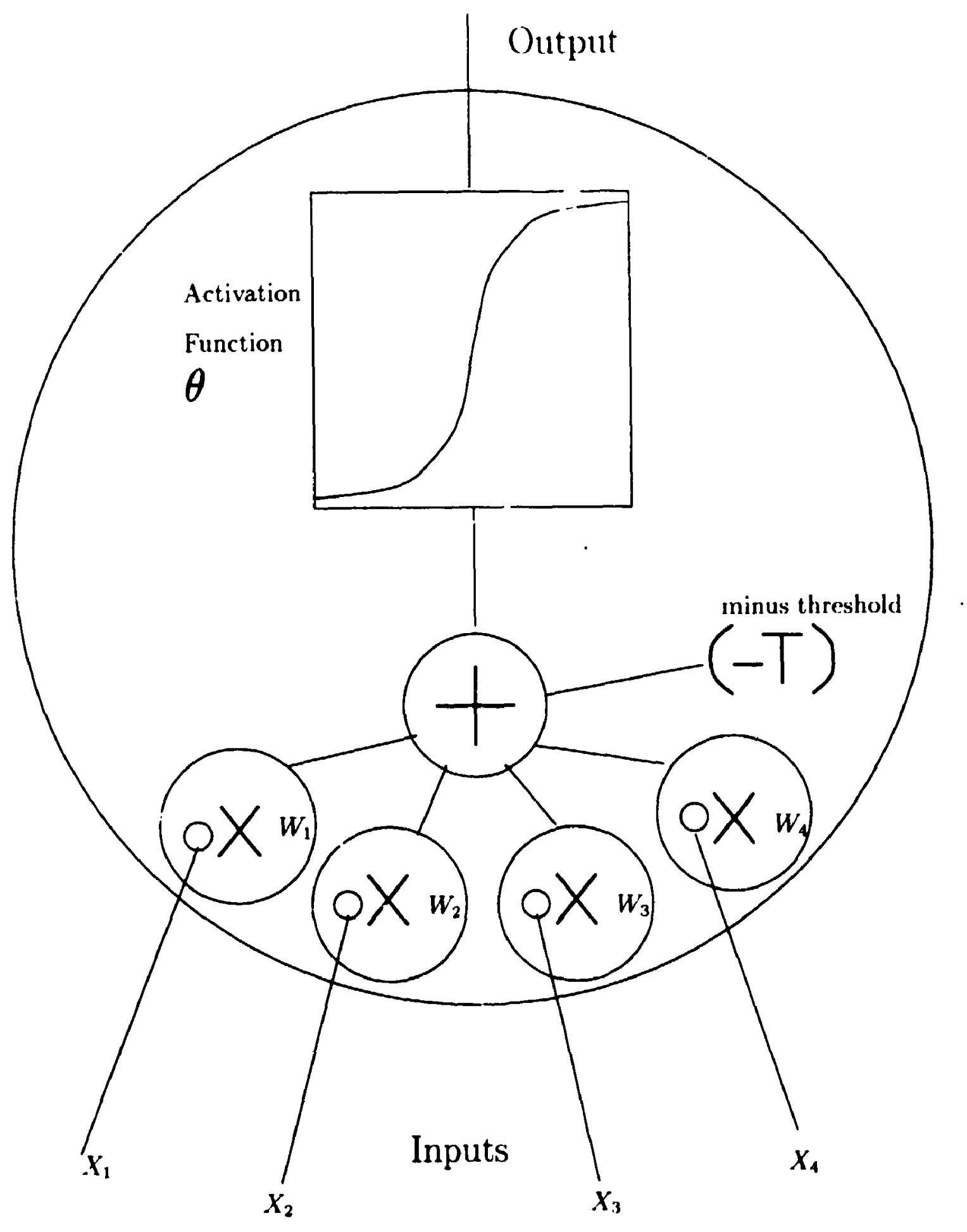

Figure 1.4: A Neural Network Node. The inputs are multiplied by weights and summed together. The sum is then passed through the activation function $\theta$. The function calculated by the node here is $\theta\left(W_{1} X_{1}+W_{2} X_{2}+W_{3} X_{3}+W_{4} X_{4}-T\right)$. 


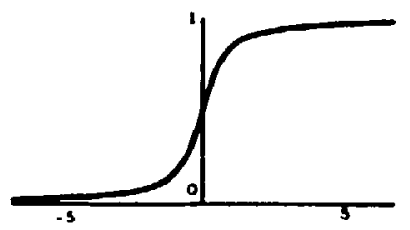

Figure 1.5: Standard sigmoid function

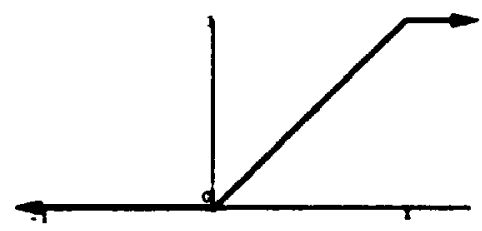

Figure 1.6: $\pi$ function

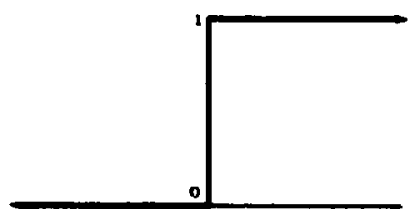

Figure 1.7: Heaviside step function 
To compare neural networks as a model of analog computation, consider a standard model of analog computation: the polynomial. Polynomials compute functions from $\Re \rightarrow \Re$. A polynomial of degree $n$ is a function $p(x)=a_{0}+a_{1} x+a_{2} x^{2}+\cdots+$ $a_{n} x^{n}$. Given a set of any $m$ points $\left\{\left(x_{1}, y_{1}\right),\left(x_{2}, y_{2}\right), \cdots,\left(x_{m}, y_{m}\right)\right\}$ it is known that there is always a polynomial $p(x)$ of degree $m-1$ such that $y_{1}=\mu\left(x_{1} ;\right.$ for all $m$ points in the point set. Furthermore, it is known that there are point sets where there does not exist a polynomial $p(x)$ of degree $m-2$ such that $y_{1}=p\left(x_{1}\right)$ for all $m$ points in the point set.

The interpolation capacity oi a class of functions $\mathcal{F}$ is defined as the size of the smallest point set over all members $f$ of $\mathcal{F}$ that can be computed. A lower bound on the interpolation capacity of a class of functions $\mathcal{F}$ means that all possible sets of points that size, and smaller, can be realized by $\mathcal{F}$. An upper bound means that there are sets of points of greater size that can not be realized by $\mathcal{F}$. For example, the interpolation capacity of the class of $n$ degree polynomials is $n+1$. That is, the known upper bound is equal to the known lower bound.

It is easy to find a polynomial to interpolate a set of points. Lagrange's polynomial interpolation formula constructs such a polynomial for the above point set.

$$
P_{n}(x)=\sum_{k=0}^{n}\left[\frac{\prod_{i=0}^{k-1}\left(x-x_{i}\right) \prod_{i=k+1}^{n}\left(x-x_{i}\right)}{\prod_{i=0}^{n-1}\left(x_{k}-x_{i}\right) \prod_{i=k+1}^{n}\left(x_{k}-x_{i}\right)} y_{k}\right]
$$

$P_{n}(x)$ is a polynomial of degree $n$ that interpolates the $n+1$ points $\left(x_{0}, y_{0}\right),\left(x_{1}, y_{1}\right), \cdots,\left(x_{n}, y_{n}\right)$. Furthermore this polynomial is unique. It is the only $n$-th degree polynomial that can fit the points.

Different terminology is used for the process of constructing a neural network to interpolate a set of data. The word interpolation is too mathematical for such a lofty class of functions that aspire to achieve human consciousness. Rather, terms such as learning a task or training are used.

It was shown by Sontag in [27] that for networks with arbitrary activation functions, the interpolation capacity of a network with $n$ inputs is the same as the 
interpolation capacity of a network with one input if they have the same node structure. The case of the one-input network interpolation capacity being contained in that of the $n$-input network is obvious. The other case of the $n$-input network interpolation capacity being contained in that of the one-input network is by showing that the weights of an $n$-input network can be set to achieve a one to one mapping of any $n$-dimensional data set to a l-dimensional data set. Under this mapping the network is equivalent to a one-input network. Thus, any bounds for one-input networks imply the same bounds for the equivalent $n$-input networks with the same node structure.

This thesis has three main results.

1. We improve the known upper bound of one layer $\theta_{s}$-networks with $k$ nodes from $3 k+1$ to $2 k$.

2. We prove a lower bound of two layer $\pi$-networks with $k$ nodes on the first layer and $d$ nodes on the second layer to be $k d$.

3. We prove a lower bound of two layer $\theta_{3}$-networks with $k$ nodes on the first layer and $d$ nodes on the second layer to be $k d$.

The proof in case 3 is actually valid for a large class of asymptotic sigmoids, with the $\theta$, being an instance of the more general theorem.

The next chapter explains why lower bounds are important to the theory of neural networks. 


\section{Chapter 2}

\section{Related Research}

\section{Learning is Hard}

To construct a polynomial that interpolates a set of points is computationally easy. Lagrange's formula can be considered as a polynomial time algorithm. Making neural networks learn a task has been shown to be a computationally difficult problem. That is, it is difficult for any algorithm to make a network interpolate' a set of data correctly. Most of the important instances are NP-complete [8]. In [1:3] Judd demonstrated the complexity of learning. Adapting his terminology, let $A$ be: a feed-forward neural network of an arbitrary architecture. Let $F$ be a configuration of network $A$, i.e., an assignment of values to the weights and thresholds of the nodes of $A$. Also let $\mathcal{M}$ be the function calculated by network $A$ with configuration $r$. Considering only boolean functions, a network with $\mathbf{n}$ inputs and 1 output computes

$$
\mathcal{M}_{F}^{A}:\{0,1\}^{n} \rightarrow\{0,1\}
$$

A task $T$ is a subset of $\left(\{0,1\}^{n},\{0,1\}\right)$. The following decision problem is $N P$ complete for neural network architectures over most types of computation nodes, including those with activation functions $\mathcal{H}, \pi, \theta_{3}$ and any bounded, monotonic function.

Instance: An architecture $A$ and a task $T$.

Question: Is there a configuration $F$ for $A$ such that $T \subseteq\left\{(\delta, \rho):\left(\mathcal{M}_{F}^{A}(\delta)\right.\right.$ agrees with $\left.\rho, \delta \in\{0,1\}^{n}, \rho \in\{0,1\}\right\}$ ? 
This implies that there does not exist, dssuming $P \neq N P$. a computationally feasible general algorithm that takes an architecture $A$ and a task $T$ and returns the proper configuration $F$ for $A$ that agrees with $T$, if such a configuration exists and returns false otherwise.

Instead of having algorithms for an arbitrary architecture and an arbitrary task. can we hope to do better by constraining the class of architectures for an algorithm that solves the above decision problem for an arbitrary task? This question can be answered for the case of networks with Heaviside activation functions $\mathcal{H}$, i.e., circuits from $\Re^{n} \rightarrow\{0,1\}$. In $[15,21]$ it is shown how the problem of separating two point sets in $n$ dimensional space by hyperplanes is equivalent to the problem of constructing a two layer $K$-net. In [19] Megiddo gives the complexity measures of this problem. The full abstract of this paper will be quoted because of its important implications to neural networks.

It is $N P$-complete to recognize whether two sets of points in general space can be separated by two hyperplanes. It is $N P$-complete to recognize whether two sets of points in the plane can be separated with $k$ lines. For every fixed $k$ in any fixed dimension, it takes polynomial time to recognize whether two sets of points can be separated with $k$ hyperplanes.

In neural network terminology, this is equivalent to the following cases.

1. It is $N P$-complete to determine if a two layer $\mathcal{H}$-net, having two nodes on the first layer and one node on the second layer, can realize any arbitrary task from $\Re^{n} \rightarrow\{0,1\}$.

2. It is $N P$-complete to determine if a two layer $\mathcal{H}$-net, having $k$ nodes on the first layer and one node on the second layer, can realize any arbitrary task from $\Re^{2} \rightarrow\{0,1\}$. 
3. It can be determined in polynomial time if a two laver $\mathcal{H}$-net, having a fixed number of nodes on the first layer and one node on the second laver with a fixed number of inputs $d$ to each node in the first laver, can realize any. arbitrar: task from $\Re^{d} \rightarrow\{0,1\}$.

An alternate proof of the first case can also be found in [?].

Not a lot is known for the above three cases when arbitrary sigmoids are used for the activation functions. The current best known result corresponds to caste I above. From $[9,10]$, it is $N P$-complete to determine if a two layer net with sigmoidal activation functions, having two nodes on the first layer and one node on the second layer, where the weights are required to be binary but the thresholds can be arbitrary rational numbers, can realize any arbitrary sized task from $\Re^{n} \rightarrow\{0,1\}$. It is also conjectured that the same problem is $N P$-complete without the above parameter constraints.

Thus, if we want computational feasible training algorithms, i.e., algorithms that run in polynomial time for arbitrary sized networks, criteria must be found that guarantee when a task can be realized by an architecture. Lower bounds on the interpolation capacity of the architecture is an important criterion, since it guarantees conditions when an architecture can realize a task.

There are other ways of getting around the $N P$-completeness of a general algorithm that trains a given network to learn a task, which is the paradigm that the back-propagation algorithm $[24,25]$ belongs to. An algorithm could grow a network. That is, if the algorithm was having problems training a network it could ard more nodes to the architecture as needed. For two opposite approaches of this strategy see $[6,20]$.

\section{Previous Results on the Interpolation Capacity of Neural Networks}

In [27] Sontag considered only one-layer networks. For a one-layer network with 
k. nodes the following results were proved for the associated activation functions:

$$
\begin{aligned}
& \mathcal{H}: \text { An exact bound of } k+1, \\
& \pi: \text { An exact bound of } 2 k \\
& 0: \text { A lower bound of } 2 k-1,
\end{aligned}
$$

where $\theta$ is any sigmoid such that there exists at least one point where it is differentable and the derivitive is not equal to zero. Obviously the standard sigmoid. $\theta_{a}$, satisfie: this condition.

The first upper bounds for a one layer network having $k$ nodes with $\theta_{\mathbf{s}}$ activation functions was given in [18] by Macintyre and Sontag as $3 k+1$. The upper bounds, even for networks with an arbitrary number of layers, is simply equal to the number of adjustable parameters the architecture has, i.e., the number of weiglits and thresholds. Thus, to determine upper bounds for multilayer $\theta_{\mathbf{s}}$-nets one needs simply to determine the total number of weights and thresholds in the network. This thesis improves the upper bound from $3 k+1$ to $2 k$ for one-layer sigmoidal networks. Unfortunately, their results do not apply to multilayer $\pi$-nets. As of yet, no known upper bounds for multilayer $\pi$-nets exist. This thesis establishes the first lower bounds.

In [17] Yamasaki and Sakurai consider the interpolation capacity of what they call $n-h-1$ networks. This is a two-layer network with $n$ inputs, $h$ nodes of the first layer, and 1 node for the output. The activation function is a slight modification of $\delta_{s}$. In effect, what they show is that if all subsets of size $n$ of the data set are linearly independent then the network has a lower bound on the interpolation capacity of $[n h / 2\rceil-1$. However, they did not realize that they could have added a layer of $n$ nodes to the bottom of the network to produce the linearly independent mapping so that they would not have to put their restriction on the data set. This is the key idea to the lower bound in this thesis for two layer asymptotic sigmoid networks. With this insight, a lower bound of $[n h / 2\rceil-1$ for a two-layer network can be achieved (keep in mind that they have an extra sigmoid at the top) with their activation function. Theorem 5.2 includes this case and improves their lower bound by a factor of 2 . 


\section{Continuous Function Approximation}

It is known that polynomials can approximate any continuous function by Weierstrass' theorem. That is. for any function $f(x)$, which is continuous in an interval I. there exists some polynomial $p(x)$ such that $|f(x)-p(x)|<$ f for all,$>0$ and for all $x \in I$.

One- and two-layer sigmoidal neural networks also can approximate any continuous function, even networks using the Heaviside activation function. Approximation results for various classes of networks have been shown by many prople $[4,5,7,11,12]$. A simple constructive proof that a one-layer network can approximate any continuous function from $\Re \rightarrow \Re$ is given in [14]. In [22] it is proved that any continuous function from $\Re^{n} \rightarrow \Re$ can be constructed by a two-layer network if the activation function is sigmoidal and there exists a point on the sigmoid where it is $\boldsymbol{n}$ times differentable and not equal to zero. 


\section{Chapter 3}

\section{An Upper Bound}

A simple theorem is given that shows that a single layer network, having $k$ nodes with the standard sigmoid $\theta$, as its activation function, has an upper bound on its interpolation capacity of $2 k$. This improves the previous best upper bound of $3 k+1$. Actualiy a more general theorem is proven, with the standard sigmoid being an instance.

The standard sigmoid is $\theta_{0}(x)=1 /\left(1+e^{-x}\right)$. Its derivative is $\theta_{g}^{\prime}(x)=(1-$ $\left.\theta_{s}(x)\right) / \theta_{s}(x)$.
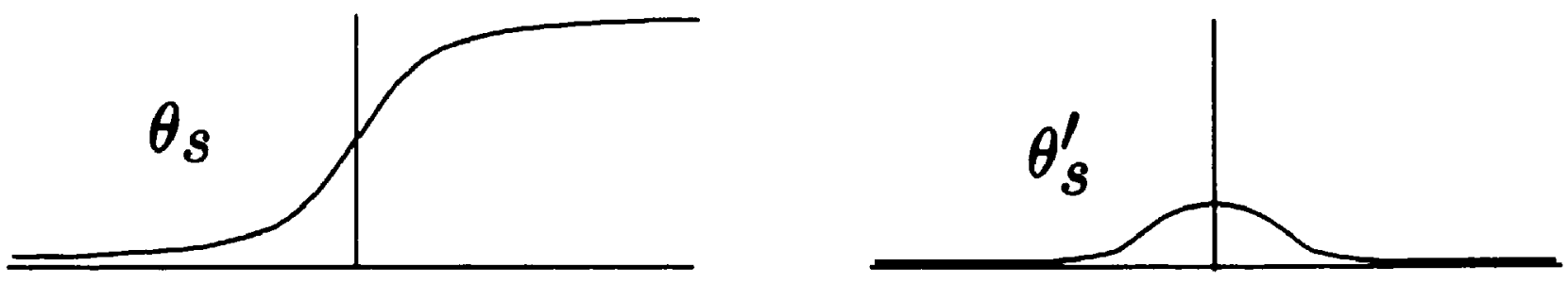

Figure 3.1: The standard sigmoid and its derivative

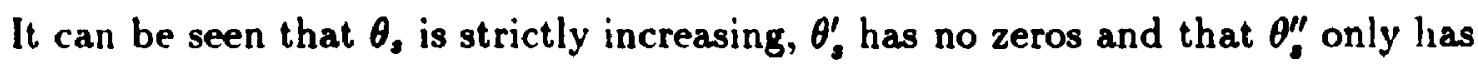
one zero.

Theorem 3.1 Any one layer neural net given by net $(x)=\sum_{i=1}^{k} a_{i} \theta\left(W_{i} x-T_{i}\right)+b$, where the activation function $\theta^{\prime}(x)$ does not equal zero for any point $x$ and $\theta^{\prime \prime}(x)=0$ at exactly one value of $x$, can interpolate at most $2 k$ points. 


\section{PROOF}

Consider a set of $n$ points $\left\{\left(x_{1}, y_{1}\right), 1 \leq i \leq n\right\}$, where $x_{1}=i$ and

$$
y_{1}= \begin{cases}y_{1}=.25 & , \text { for } i \text { odd } \\ y_{1}=.75 & , \text { for } i \text { even }\end{cases}
$$

See, figure 3.2.

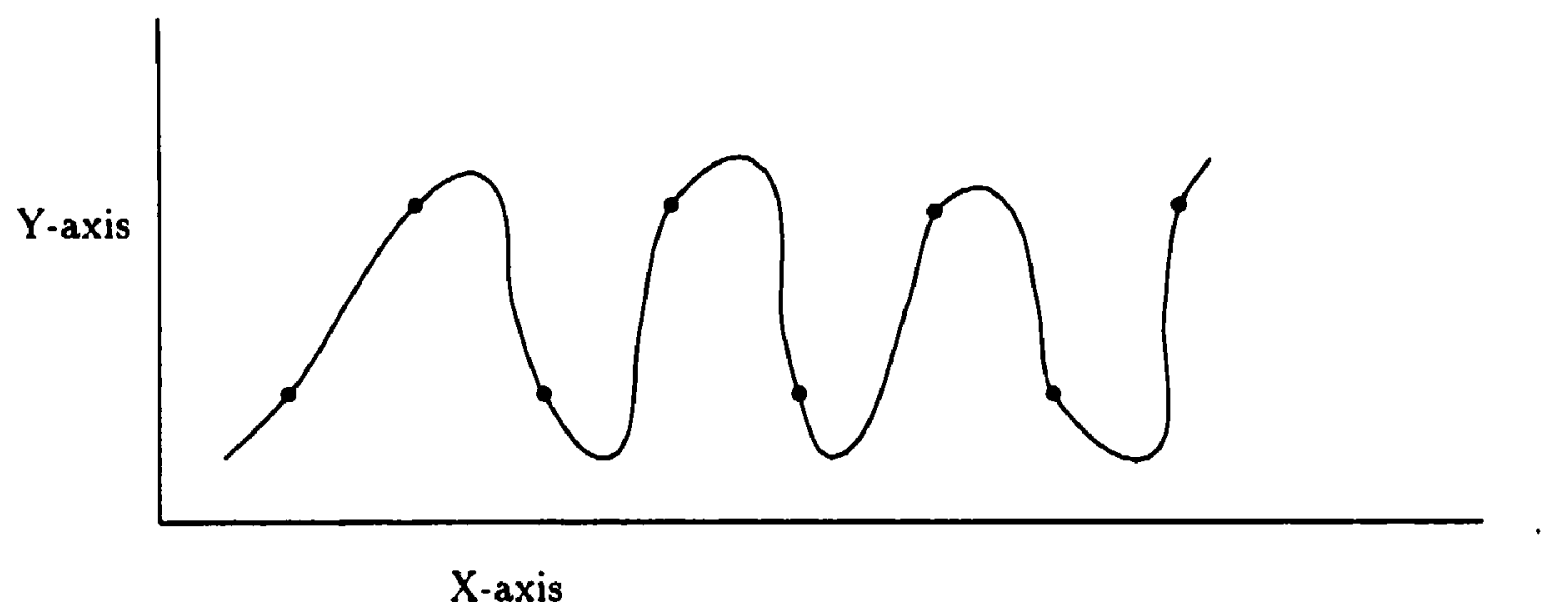

Figure 3.2: A difficult point set for a one layer net

It is clear that any continuous curve that interpolates $n$ of these points must have at least $n-2$ zeros of the first derivative, by applying the mean value theorem $n-2$ times.

The $n$ points must be interpolated with a one layer network given as net $(x)=$ $\sum_{i=1}^{k} a_{i} \theta_{9}\left(W_{i} x-T_{i}\right)+b$ and its derivative net' $(x)=\sum_{i=1}^{k} a_{i} W_{1} \theta_{9}^{\prime}\left(W_{1} x-T_{3}\right)$ must have at least $n-2$ zeros.

Consider a class of functions $f_{1}(x)$ where $f_{2}^{\prime}(x)=0$ for one value of $x$, say $x_{0}$. If to the left of $x_{0}$ it is strictly increasing then to the right of $x_{0}$ it must be strictly decreasing. Alternatively, if to the left of $x_{0}$ it is strictly decreasing then to the right of $x_{0}$ it must be strictly increasing. Either way, it is obvious that for a value $y_{1}$ there can only be at most two points, say $x_{1}$ and $x_{2}$, where $y_{1}=f\left(x_{1}\right)$ and $y_{1}=f\left(x_{2}\right)$. It is also clear that if we take a linear combination of $k$ of these functions then there 
call be at most $2 k$ values of $x$ where the resulting linear combination has the same value for $y$. That is, assuming that the $a_{1}$ 's are not zero

$$
\sum_{i=1}^{k} a_{i} f_{i}(x) \text { can have the same value } 2^{\prime} \text { most } 2 k \text { times. }
$$

However, if none of the $k$ functions, from the linear combination, has the value 0 for any $x$ then there can only be at most $2(k-1)$ values of $x$ for which this linear combination has the value 0 . This follows because a non zero scalar multiple of a function without a zero can not result in a function with a zero. If only two multiples of these functions are added then the sum can only have at most two points where they are zero because at no point are the individual functions zero and they are strictly increasing and decreasing on each side of the zeros of their derivatives. When the other $k-2$ scalar multiples are added, use fact (3.1) again on the smaller case to get at most $2 k-2$ zeros.

\footnotetext{
$\sum_{i=1}^{k} a_{i} W_{1} \theta_{3}^{\prime}\left(W_{1} x-T_{1}\right)$ is an instance of the above class of functions. Since net $(x)=$ $\sum_{i=1}^{k} a_{3} \theta\left(W_{1} x-T_{1}\right)+b \operatorname{can}$ have at most $2(k-1)$ zeros and the point set that we want to interpolate needs $n$ zeros for $n+2$ points, net $(x)$ can only only interpolate $2 k$ of these points.
} 


\section{Chapter 4}

\section{$\pi$ Bounds}

A lower bound for the interpolation capacity of a two-layer $\pi$-net is given. It is proven by formally giving a construction that interpolates the necessary number of arbitrary data points. Recall that the piecewise linear sigmoid $\pi(x)$ is defined as

$$
\pi(x)= \begin{cases}0 & \text { if } x \leq 0 \\ 1 & \text { if } x \geq 1 \\ x & \text { otherwise }\end{cases}
$$

The two-layer network, $\pi$-net $(x): \Re \rightarrow \Re$, can be expressed algebraically as

$$
\pi-\operatorname{net}(x)=\sum_{j=1}^{d}\left[W, \pi\left(\sum_{i=1}^{k}\left[W_{i, j}^{\prime \prime} \pi\left(W_{i}^{\prime} x-T_{\imath}^{\prime}\right)\right]-T_{j}^{\prime \prime}\right)\right]-T,
$$

where the $W$ 's, $W^{\prime}$ 's, and $W^{\prime \prime}$ 's correspond to the weights and the $T$ ' 's, $T^{\prime \prime}$ 's, and $T^{\prime \prime}$ 's correspond to the thresholds. In figure 4.1 the corresponding weights and thresholds are labeled as edges on, the graph. The $W^{\prime}$ 's and the $T^{\prime \prime}$ 's represent the weights and thresholds of the nodes on the first level, the $W^{\prime \prime}$ 's and the $I^{\prime \prime \prime}$ 's represent the weights and thresholds of the nodes on the second level respectively, and the $W$ 's and $T$ represent the weights and threshold, respectively, used in the final sum of the outputs of the second layer.

The basic idea behind the proof is that for each point to be interpolated only one node from the first layer and one node from the second layer need be considered. In this way we can avoid the complex interactions between nodes that typically urcur 


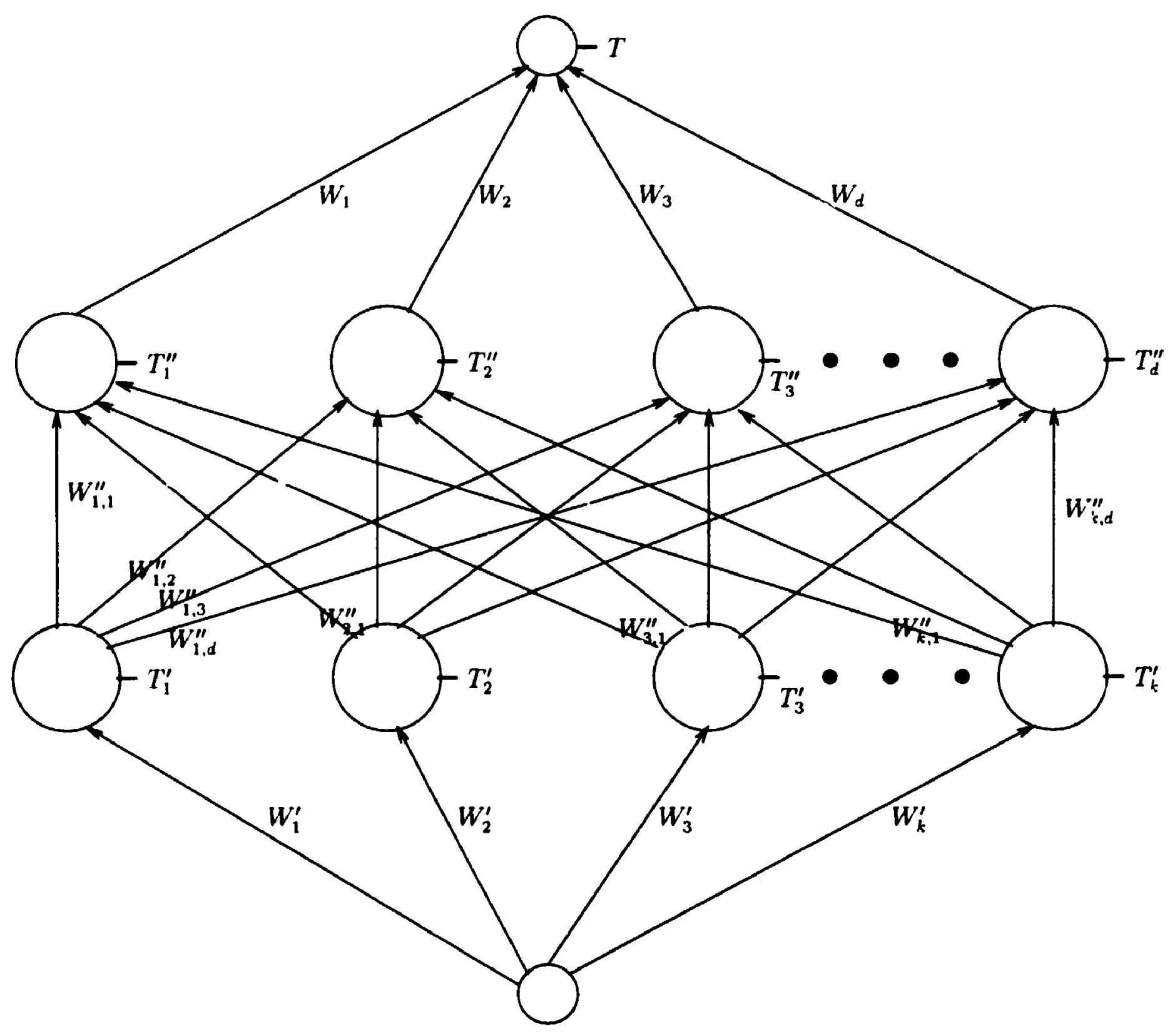

Figure 4.1: Weight assignments of the network 
in neural networks. To this end. we must determine conditions for when we can disregard this interaction. Hence, the following definition.

Definition: The region of activation of a function is the part of the domain with non zero slope. That is, a change in the domain will result in a change in the range. For $\pi\left(\because{ }^{\prime}\right.$ the region of activation is $0<x<1$.

What we .. ant to prove is the following theorem.

Theorem 4.1 A two-layer network with $\pi$-activation functions, haling $k$ nodes on the first layer and $d$ nodes of the second layer, can interpolate any kd points.

\section{Proof}

The $k d$ points will be labeled as

$$
p_{i, j}=\left(x_{i, j}, y_{i, j}\right): 1 \leq i \leq k, 1 \leq j \leq d
$$

with

$$
x_{1,1}<x_{1,2}<\cdots<x_{1, d}<x_{2,1}<x_{2,2}<\cdots<x_{2, d}<\cdots<x_{k, 1}<x_{k, 2}<\cdots<x_{k, d}
$$

The standard assumption will be made that no two points can have the same $x$ value. Since by the definition of a function, if two points have the same $x$ value and different $y$ values then the two points can not be realized by any function. See figure 4.2 .

The proof will be by giving a construction of the required network. Here is an outline of the main steps in the proof.

Step 1 The first layer will have $k$ nodes that divide the $X$-axis into $k$ disjoint regions of activation. Thus for a value $x$, only one region in the first level of the network will be active. Each region will have $d$ of the points to be interpolated. We will show how to assign the weights and the thresholds to the nodes in the first level to achieve this.

Step 2 As a result of step 1, only one of the $k$ inputs to the nodes of the second level will be active in a given region. All the other inputs can be regarded as constants within this region. 


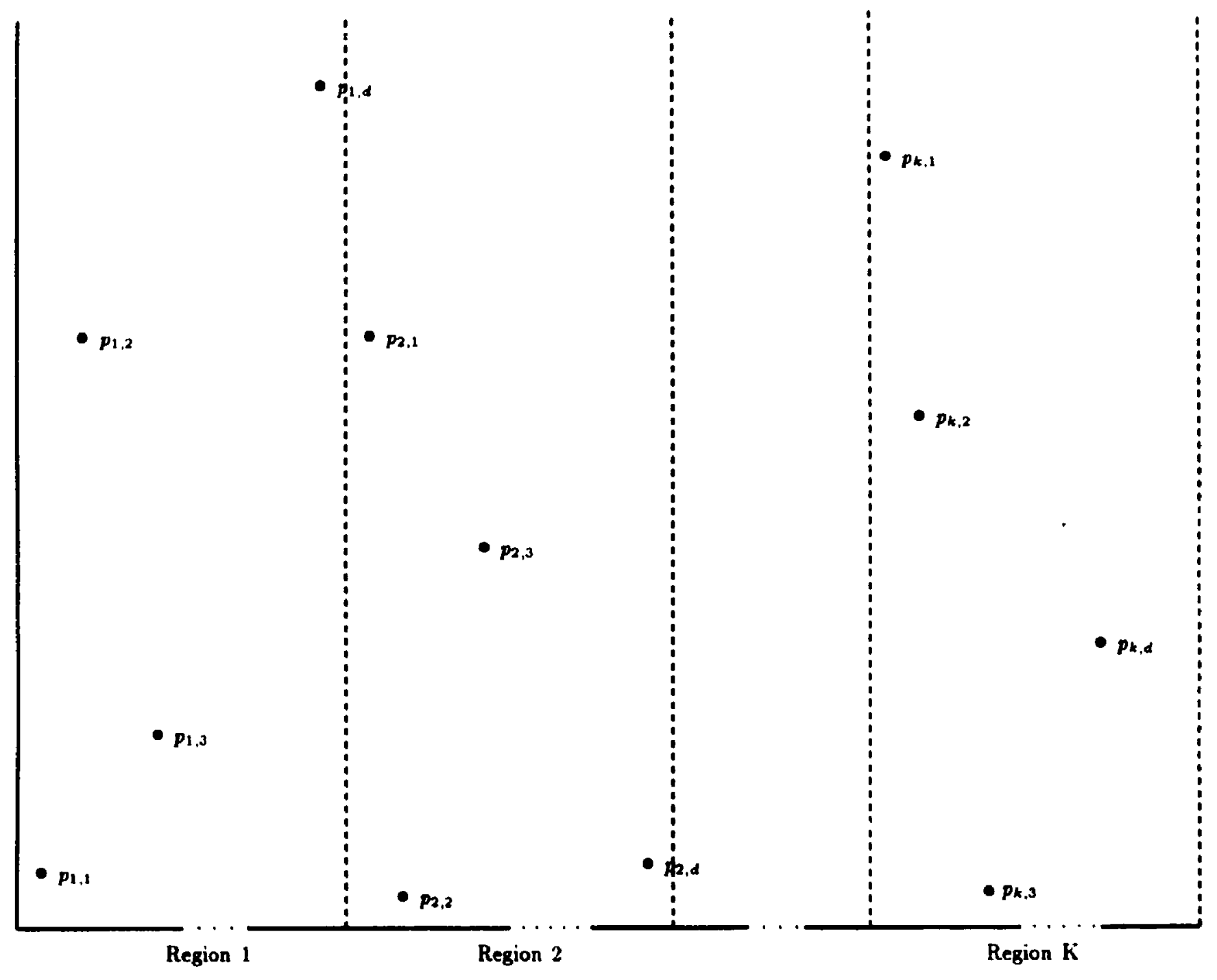

Figure 4.2: The Points of interpolation. The first layer of the network will divide the $\mathrm{X}$-axis into $\mathrm{K}$ regions; each node will represent one region. Each node in the second layer will interpolate $K$ points, one point from each of the $K$ regions. 
Step 3 Each node in the second layer will interpolate exactly one point from each of the $k$ regions. For node $j$ of the second layer these points will be $\left(x_{1, j}, y_{1, J}\right),\left(x_{2, J}, y_{2, J}\right) \ldots\left(x_{k, J}, y_{k, J}\right)$.

Step 1 Relying on step 2. we show how to assign the weights to nodes on the second level to achieve the claim of step 3.

Step 5 For each point that is interpolated, only one node in the second layer will be active for a region that includes that point, and all other nodes in the second layer will be constant for that region. We will show how to assign the thresholds in the second layer of the network to achieve this.

Step 6 Finally, we show how to assign the weights that sum the outputs of the nodes on the second level that achieve the final desired interpolation of all the $k$ a points.

\section{The Construction}

Step 1 First we have to choose $k$ disjoint regions with $d$ points in each region. The region intervals will be Region ${ }_{i}=\left[a_{i}, b_{i}\right]: 1 \leq i<k$. Choose $\left[a_{1}, b_{1}\right]$ with $\left\{\begin{array}{lll}a_{1}<x_{1,1} & \text { and } x_{1, d}<b_{1}<x_{2,1} & \text { for Region }_{1} \\ b_{i-1}<a_{i}<x_{i, 1} & \text { and } x_{i, d}<b_{i}<x_{i+1,1} & \text { for Region } \\ 1 & : 2<i<k-1 \\ b_{k-1}<a_{i}<x_{k, 1} & \text { and } x_{k, d}<b_{i} & \text { for Region }\end{array}\right.$ as the required regions. Now set $W_{i}^{\prime}=\frac{1}{b_{1}-a_{1}}$ and $T_{i}^{\prime}=\frac{a_{1}}{b_{1}-a_{1}}$ for the nodes in the first level. It follows from lemma 4.1 that the nodes will have regions of activation $\left[a_{1}, b_{1}\right]$. They are also disjoint because they were chosen to be so.

LEMMA 4.1 For any real numbers $a$ and $b$ such that $a<b$, the function

$$
\phi(x)=\pi\left(\left(\frac{1}{b-a}\right) x-\frac{a}{b-a}\right)
$$

is given by

$$
\phi(x)= \begin{cases}0 & \text { if } x \leq a \\ 1 & \text { if } x \geq b \\ \frac{x-a}{b-a} & \text { otherwise }\end{cases}
$$


ProOF

First observe that

$$
\phi(x)=\pi\left(\left(\frac{1}{b-a}\right) x-\frac{a}{b-a}\right)=\pi\left(\frac{x-a}{b-a}\right) .
$$

Then we have the following three cases.

Case $1 \phi(x)=0$ when $\frac{x-a}{b-a} \leq 0$. This happens when $x \leq a$.

Case $2 \phi(x)=1$ when $\frac{x-a}{b-a} \geq 1$. This happens when $x \geq b$.

Case 3 For $a<x<b$ then $0<\frac{x-a}{x-b}<1$ from the two case above. Thus $\phi(x)=\frac{x-a}{b-a}$.

Step 2 Each node in the second layer receives as input a weighted output from each of the $k$ nodes in the first layer. Since the inputs to the nodes on the second layer represent disjoint activation regions, at most one of the inputs to each of them will be active. All the other inputs can be regarded as constants within this region. Let $Y_{i}=\pi\left(W_{i}^{\prime} X-T_{i}^{\prime}\right)$ be the output of node ${ }_{i}$. From the above observation and lemma 4.1 it follows that

$$
Y_{i}= \begin{cases}1 & \text { if } x_{h, j} \text { is in region }{ }_{h}, \text { for } h<i \\ \frac{x_{i, y}-a_{i}}{b_{1}-a_{1}} & \text { if } x_{h, i} \text { is in region } \\ 0 & \text { if } x_{h, j} \text { is in region }{ }_{h}, \text { for } h>i\end{cases}
$$

Step 3 Each node in the second layer will interpolate exactly one point from each of the $\mathrm{k}$ regions. For node $j$ of the second layer these points will be $\left(x_{1, \jmath}, y_{1, j}\right),\left(x_{2, j}, y_{2, j}\right), \ldots\left(x_{k, j}, y_{k, j}\right)$.

Step 4 Relying on step 2 we show how to assign the weights to nodes on the second level to achieve step 3 . Let $Y_{i, j}=\frac{x_{i, j}-a_{i}}{b_{1}-a_{i}}$. Since each of the $k$ points come from $d$ different regions, we have the output $Z$, of node $j$ for the $k$ points being

$$
\begin{aligned}
& Z_{1, J}=\pi\left(W_{1, J}^{\prime \prime} Y_{1, j}-T_{,}^{\prime \prime}\right) \\
& Z_{2, J}=\pi\left(W_{1, j}^{\prime \prime}+W_{2, j}^{\prime \prime} Y_{2, j}-T_{j}^{\prime \prime}\right) \\
& Z_{3, J}=\pi\left(W_{1, j}^{\prime \prime}+W_{2, j}^{\prime \prime}+W_{3, j}^{\prime \prime} Y_{3, j}-T_{,}^{\prime \prime}\right)
\end{aligned}
$$




$$
Z_{k, J}=\pi\left(W_{1, J}^{\prime \prime}+W_{2, j}^{\prime \prime}+\cdots+W_{k-1, J}^{\prime \prime}+W_{k, J}^{\prime \prime} Y_{k, J}-T_{\jmath}^{\prime \prime}\right)
$$

or in general form

$$
Z_{i, J}=\pi\left(\sum_{h=1}^{i-1} W_{h, J}^{\prime \prime}+W_{i, J}^{\prime \prime} Y_{t, J}-T_{\jmath}^{\prime \prime}\right), 1 \leq i \leq k
$$

Now we will make the assumption that $0<Z_{\mathbf{t}}<1$. If this is the case then $0<$ $\sum_{h=1}^{i-1} W_{h, J}^{\prime \prime}+W_{i, J}^{\prime \prime} Y_{i, J}-T_{\jmath}^{\prime \prime}<1$, which follows from our definition of the $\pi$ function. Thus, under this assumption it follows that

$$
Z_{i, j}=\sum_{h=1}^{i-1} W_{h, j}^{\prime \prime}+W_{i, j}^{\prime \prime} Y_{i, j}^{\prime}-T_{j}^{\prime \prime}
$$

Soiving for $W_{\mathrm{\imath}, \mathrm{g}}^{\prime \prime}$ gives

$$
W_{i, J}^{\prime \prime}=\frac{Z_{i, j}+T_{j}^{\prime \prime}-\sum_{h=1}^{i-1} W_{h, J}^{\prime \prime}}{Y_{i, J}}
$$

It follows from lemma 4.3 that $W_{i, j}^{\prime \prime}$ has the general solution

$$
W_{i, J}^{\prime \prime}=\frac{\sum_{h=1}^{i}\left((-1)^{i+h} Z_{h, J} \prod_{g=h+1}^{i-1}\left(1-Y_{g, j}\right) \prod_{g=1}^{h-1} Y_{g, J}\right)+(-1)^{i-1} T \prod_{g=1}^{i-1}\left(1-Y_{g, J}\right)}{\prod_{g=1}^{n} Y_{g, j}}
$$

Thus if we know the $Y_{i, j}$ 's and the $Z_{i, j}$ 's and the $T$,'s we can determine the weights of all $j$ nodes of the second layer. The $Y_{i, j}$ 's were already determined in step 2. The determination of the $T$,'s will come in step 5 and the determination of the $Z_{i, j}$ 's will come from step 6 .

In the following two lemmas, lemma 4.2 is merely a helper needed for lemma 4.3. Note that for $t<s, \prod_{\mathfrak{s}}^{t}=1$ and $\sum_{s}^{t}=0$.

\section{LEMMA 4.2}

$$
(-1)^{n} \prod_{i=1}^{n}\left(1-Y_{i}\right)=\sum_{k=0}^{n}\left[(-1)^{k} \prod_{i=1}^{k-1}\left(1-Y_{i}\right) \prod_{i=k+1}^{n} Y_{i}\right]
$$


ProOf

The proof will be by weak induction.

Basis: When $n=1$

$$
\sum_{k=0}^{1}\left[(-1)^{k} \prod_{i=1}^{k-1}\left(1-Y_{i}\right) \prod_{i=k+1}^{1} Y_{i}\right]=Y_{1}-1=(-1)^{1}\left(1-Y_{1}\right)
$$

Inductive Step: Assume (4.2) holds for $n-1$, show that it holds for $n$.

$$
\begin{gathered}
\sum_{k=0}^{n}\left[(-1)^{k} \prod_{i=1}^{k-1}\left(1-Y_{i}\right) \prod_{i=k+1}^{n} Y_{i}\right]=\sum_{k=0}^{n-1}\left[(-1)^{k} \prod_{i=1}^{k-1}\left(1-Y_{i}\right) \prod_{i=k+1}^{n} Y_{i}\right]+(-1)^{n} \prod_{i=1}^{n-1}\left(1-Y_{i}\right) \\
=Y_{n} \sum_{k=0}^{n-1}\left[(-1)^{k} \prod_{i=1}^{k-1}\left(1-Y_{i}\right) \prod_{i=k+1}^{n-1} Y_{i}\right]+(-1)^{n} \prod_{i=1}^{n-1}\left(1-Y_{i}\right)
\end{gathered}
$$

Now apply the inductive hypothesis assuming that (4.2) holds for $n-1$.

$=Y_{n}(-1)^{n-1} \prod_{i=1}^{n-1}\left(1-Y_{i}\right)+(-1)^{n} \prod_{i=1}^{n-1}\left(1-Y_{i}\right)=(-1)^{n}\left(1-Y_{n}\right) \prod_{i=1}^{n-1}\left(1-Y_{i}\right)=(-1)^{n} \prod_{i=1}^{n}\left(1-Y_{i}\right)$

LEMMA 4.3 The following recursive equation

$$
W_{n}=\frac{Z_{n}+T-W_{1}-W_{2}-\cdots-W_{n-1}}{Y_{n}}=\frac{Z_{n}+T-\sum_{k=1}^{n-1} W_{k}}{Y_{n}}
$$

for $n \geq 1$, has the solution

$$
W_{n}=\frac{\sum_{j=1}^{n}\left((-1)^{n+j} Z_{j} \prod_{i=j+1}^{n-1}\left(1-Y_{i}\right) \prod_{i=1}^{j-1} Y_{i}\right)+(-1)^{n-1} T \prod_{i=1}^{n-1}\left(1-Y_{i}\right)}{\prod_{i=1}^{n} Y_{i}}
$$


ProOF

The proof will be by strong induction.

Basis: When $n=1,(4.3)$ evaluates to $\frac{Z_{n}+T}{f_{1}}$.

Inductive Step: Inductive step (strong). Assuming ( $\forall i: i<n) \| l$, holds. show that $W_{n}$ holds.

We get

$$
\begin{aligned}
& W_{n}=\frac{Z_{n}+T-\sum_{k=1}^{n-1}\left[\frac{\sum_{j=1}^{k}\left((-1)^{k+1} z, \prod_{i=j+1}^{k-1}\left(1-Y_{i}\right) \prod_{i=1}^{j-1} r_{i}\right)+(-1)^{k-1} T \prod_{i=1}^{k-1}\left(1-Y_{i}\right)}{\prod_{i=1}^{k} r_{i}}\right]}{Y_{n}} \\
& =\frac{Z_{n}}{Y_{n}}+\frac{T}{Y_{n}}-\frac{1}{Y_{n}} \sum_{k=1}^{n-1} \sum_{j=1}^{k}\left[\frac{(-1)^{k+j} Z_{j} \prod_{i=j+1}^{k-1}\left(1-Y_{i}\right) \prod_{i=1}^{j-1} Y_{i}}{\prod_{i=1}^{k} Y_{i}}\right]-\frac{T}{Y_{n}} \sum_{k=1}^{n-1}\left[\frac{(-1)^{k-1} \prod_{i=1}^{k-1}\left(1-Y_{i}\right)}{\prod_{i=1}^{k} Y_{i}}\right] . \\
& =\frac{Z_{n}}{Y_{n}}-\frac{1}{Y_{n}} \sum_{k=1}^{n-1} \sum_{j=1}^{k}\left[\frac{(-1)^{k+1} Z, \prod_{i=j+1}^{k-1}\left(1-Y_{i}\right) \prod_{i=1}^{j-1} Y_{i}}{\prod_{i=1}^{k} Y_{1}}\right]+\frac{T}{Y_{n}}\left(1-\sum_{k=1}^{n-1}\left[\frac{(-1)^{k-1} \prod_{i=1}^{k-1}\left(1-Y_{i}\right)}{\prod_{i=1}^{k} Y_{i}}\right]\right) \\
& =\frac{Z_{n}}{Y_{n}}-\frac{1}{Y_{n}} \sum_{k=1}^{n-1} \sum_{j=1}^{k}\left[\frac{(-1)^{k+j} Z_{j} \prod_{i=j+1}^{k-1}\left(1-Y_{i}\right) \prod_{i=1}^{j-1} Y_{i}}{\prod_{i=1}^{k} Y_{i}}\right]-\frac{T}{Y_{n}} \sum_{k=0}^{n-1}\left[\frac{(-1)^{k-1} \prod_{i=1}^{k-1}\left(1-Y_{i}\right)}{\prod_{i=1}^{k} Y_{i}}\right] \\
& =\frac{Z_{n}}{Y_{n}}-\frac{1}{Y_{n}} \sum_{k=1}^{n-1} \sum_{j=1}^{k}\left[\frac{(-1)^{k+1} Z_{j} \prod_{i=j+1}^{k-1}\left(1-Y_{i}\right) \prod_{i=1}^{j-1} Y_{i} \prod_{i=k+1}^{n-1} Y_{i}}{\prod_{i=1}^{n-1} Y_{i}}\right]+\frac{T}{Y_{n}} \sum_{k=0}^{n-1}\left[\frac{(-1)^{k} \prod_{i=1}^{k-1}\left(1-Y_{i}\right) \prod_{i=k+1}^{n-1} Y_{i}}{\prod_{i=1}^{n-1} Y_{i}}\right]
\end{aligned}
$$


Now using the standard technique of changing the order of summations, for any function $f(x, y)$ we have $\sum_{i=1}^{s} \sum_{j=1}^{1} f(i, j)=\sum_{j=1}^{s} \sum_{i=j}^{s} f(i, j)$.

$=\frac{Z_{n}}{Y_{n}}-\frac{1}{Y_{n}} \sum_{j=1}^{n-1} \sum_{k=1}^{n-1}\left[\frac{(-1)^{k+j} Z, \prod_{i=j+1}^{k-1}\left(1-Y_{i}\right) \prod_{i=1}^{j-1} Y_{i} \prod_{i=k+1}^{n-1} Y_{i}}{\prod_{i=1}^{n-1} Y_{i}}\right]+\frac{T}{Y_{n}} \sum_{k=0}^{n-1}\left[\frac{(-1)^{k} \prod_{i=1}^{k-1}\left(1-Y_{i}\right) \prod_{i=k+1}^{n-1} Y_{i}}{\prod_{i=1}^{n-1} Y_{i}}\right]$

$$
\begin{array}{r}
=\frac{Z_{n} \prod_{i=1}^{n-1} Y_{i}}{\prod_{i=1}^{n} Y_{i}}-\frac{1}{\prod_{i=1}^{n} Y_{i}} \sum_{j=1}^{n-1}\left(Z, \prod_{i=1}^{j-1} Y_{i} \sum_{k=1}^{n-1}\left[(-1)^{k+j} \prod_{i=j+1}^{k-1}\left(1-Y_{i}\right) \prod_{i=k+1}^{n-1} Y_{i}\right]\right) \\
+\frac{T}{\prod_{i=1}^{n} Y_{i}^{k}} \sum_{k=0}^{n-1}\left[(-1)^{k} \prod_{i=1}^{k-1}\left(1-Y_{i}\right) \prod_{i=k+1}^{n-1} Y_{i}\right]
\end{array}
$$

$=\frac{-\sum_{j=1}^{n}\left(Z, \prod_{i=1}^{j-1} Y_{i} \sum_{k=1}^{n-1}\left[(-1)^{k+1} \prod_{i=j+1}^{k-1}\left(1-Y_{i}\right) \prod_{i=k+1}^{n-1} Y_{i}\right]\right)+T \sum_{k=0}^{n-1}\left[(-1)^{k} \prod_{i=1}^{k-1}\left(1-Y_{i}\right) \prod_{i=k+1}^{n-1} Y_{i}\right]}{\prod_{i=1}^{n} Y_{i}}$

$=\frac{\sum_{j=1}^{n}\left(Z, \prod_{i=1}^{j-1} Y_{i} \sum_{k=1}^{n-1}\left[(-1)^{k+j+1} \prod_{i=j+1}^{k-1}\left(1-Y_{i}\right) \prod_{i=k+1}^{n-1} Y_{i}\right]\right)+T \sum_{k=0}^{n-1}\left[(-1)^{k} \prod_{i=1}^{k-1}\left(1-Y_{i}\right) \prod_{i=k+1}^{n-1} Y_{i}\right]}{\prod_{i=1}^{n} Y_{i}}$

We now can apply lemma (4.2) in two different places in the above equation.

$$
=\frac{\sum_{j=1}^{n}\left(Z ; \prod_{i=1}^{j-1} Y_{i}(-1)^{n+j} \prod_{j+1}^{n-1}\left(1-Y_{i}\right)\right)+T(-1)^{n-1} \prod_{i=1}^{n-1}\left(1-Y_{i}\right)}{\prod_{i=1}^{n} Y_{i}}
$$

Step 3 For each point that is interpolated, only one node in the second layer will be active for a region that includes that point, and all other nodes in the second 
layer will be constant for that region. We will show how to assign the thresholds in the second layer of the network to achieve this.

Assuming point $p_{i, j}$ is in the region of activation for node $j$ of the second level. we need to have $p_{1 . j}$ outside the region of activation of all the other nodes of the second level. That is, for each point $p_{i, j}$ none of the regions of activation of nodes in the seco:nd level can overlap. Because of the nature of our construction in step 4. $p_{i, j}$ is in the region of activation of node $j$. We just need to make the region that contains this point suitably small so as not to contain other points. If the region of activation for all nodes in the second level for all the points is less than the smallest distance between neighboring $Y_{i, j}$ points then none of the activation regions of the second level will overlap for each pair of points. The smallest distance $\Delta Y$ between the $Y_{i, j}$ 's will be less than or equal to the smallest distance, $\Delta . X$, between $x_{i, j}$ 's multiplied by the smallest of the weights of the nodes of the first level.

$$
\begin{gathered}
\Delta Y=\min _{1 \leq i \leq k-1,1 \leq j \leq d-1,1 \leq g \leq i, 1 \leq h \leq j} Y_{i, j}-Y_{g, h} \\
\leq \min _{1 \leq i \leq k} W_{l}^{\prime} \min _{1 \leq i \leq k-1,1 \leq j \leq d-1,1 \leq g \leq i, 1 \leq h \leq j} X_{i, j}-X_{g, h}=\Delta X
\end{gathered}
$$

It follows from lemma 4.4 that for all weights $W_{i, j}$, we need $\frac{1}{\left|W_{1, j}\right|}<\Delta . X$ or $\left|W_{i, j}\right|>\frac{1}{\Delta X}$.

LEMMA 4.4 The width of the region of activation of $\pi(A X-B)$ is $\frac{1}{|A|}$.

Proof

$\pi(A X-B)=0$ when $A X-B \leq 0$ or equivalently when $X \leq|A|$.

$\pi(A X-B)>0$ when $A X-B \geq 1$ or equivalently when $X \geq \frac{1+B}{|A|}$.

Thus the region of activation is $\left[\frac{B}{|A|}, \frac{1+B}{|A|}\right]$ and the width of the activation region is $\frac{1+B}{|A|}-\frac{B}{|A|}=\frac{1}{|A|}$.

It follows from lemma 4.5 that $\left|W_{i, y}\right|>\frac{1}{\Delta X}$ occurs when

$$
T_{,}^{\prime}>\frac{2 \Delta X \prod_{g=1}^{i} Y_{b}}{\prod_{g=1}^{i-1}\left(1-Y_{g}\right)}, \text { for all } 1 \leq j \leq d .
$$




\section{LEMMA 4.5}

$$
\begin{gathered}
\text { If } W_{n}=\frac{\sum_{j=1}^{n}\left((-1)^{n+1} Z_{j} \prod_{i=j+1}^{n-1}\left(1-Y_{i}\right) \prod_{i=1}^{j-1} Y_{i}\right)+(-1)^{n-1} T \prod_{i=1}^{n-1}\left(1-Y_{i}\right)}{\prod_{i=1}^{n} Y_{i}} \\
\text { and } T>\frac{2 V \prod_{i=1}^{n} Y_{i}}{\prod_{i=1}^{n-1}\left(1-Y_{i}\right)} \text { then }\left|W_{n}\right|>V .
\end{gathered}
$$

\section{ProOF}

$$
\begin{aligned}
& \text { Let } A=\sum_{j=1}^{n}\left((-1)^{n+1} Z_{j} \prod_{i=j+1}^{n-1}\left(1-Y_{i}\right) \prod_{i=1}^{j-1} Y_{i}\right) \text { and } T=\frac{2 A B}{\prod_{i=1}^{n-1}\left(1-Y_{i}\right)} \text { for some } B . \\
& \text { Thus for }\left|W_{n}\right|=\left|\frac{A+(-1)^{n-1} 2 A B}{\prod_{i=1}^{n} Y_{i}}\right| \geq \frac{|A B|}{\prod_{i=1}^{n} Y_{i}} \text { to be greater ii:an } V, \\
& \text { we need } B>\frac{V \prod_{i=1}^{n} Y_{i}}{A} \text {. Which gives } T>\frac{2 V \prod_{i=1}^{n} Y_{i}}{\prod_{i=1}^{n-1}\left(1-Y_{i}\right)} .
\end{aligned}
$$

Step 6 Finally, we show how to assign the weights that sum the outputs of the nodes on the second level that achieve the final desired interpolation of all the $k d$ points. Assume for now that for the points $p_{i, j}=\left(x_{i, j}, y_{i, j}\right)$, the $y_{i, j}$ 's are greater than or equal to zero.

For the $k d$ points $p_{i, j}=\left(x_{i, j}, y_{i, j}\right)$, let $\gamma$ be the maximum $y_{i, j}$ value. That is,

$$
\gamma=\max _{1 \leq i \leq k-1,1 \leq j \leq d-1} y_{i, j}
$$

The weights $W_{,}, 1 \leq j \leq d$ will be set as $W_{j}=(-1)^{j+1} \gamma$. That is, they will alternately be assigned the values $+\gamma$ and $-\gamma$ 
Now we have to show from these weight assignments how to determine the $Z_{1, j}$ 's, deferred $^{1}$ from step 4 , so that $y_{i, j}=W, Z_{i, j}$. Let $\zeta_{j}$ equal the output of node $j$ on the second level. We have

$$
\pi-\operatorname{net}(x)=W_{1} \zeta_{1}+W_{2} \zeta_{2}+\cdots+W, \zeta_{j}=\sum_{j=1}^{d} W_{j, \zeta_{j}}=\sum_{j=1}^{d}(-1)^{j+1} \gamma_{j} .
$$

However, from step 5 only one node is active at a time. For point $p_{i, d}$

$$
\zeta_{h}= \begin{cases}1 & \text { if } h<j \\ Z_{i, j} & \text { if } h=j \\ 0 & \text { if } h>j\end{cases}
$$

Thus for point $p_{i, j}$, we have

$$
\pi-\operatorname{net}\left(x_{i, j}\right)=y_{i, j}=\sum_{h=1}^{j-1}(-1)^{h+1} \gamma+(-1)^{\jmath+1} \gamma Z_{i, j}
$$

Thus the $Z_{i, j}$ 's are set as

$$
Z_{i, j}=(-1)^{j+1}\left(\frac{y_{i, j}}{\zeta}-\sum_{h=1}^{j-1}(-1)^{h+1}\right)
$$

or equivalently as

$$
Z_{i, j}=\left\{\begin{array}{cl}
\frac{y_{i, 1}}{\zeta} & \text { if } j \text { is even } \\
1-\frac{y_{i, 2}}{\zeta} & \text { if } j \text { is odd }
\end{array}\right.
$$

Now we must take care of the case when the $y_{i, j}$ 's can be negative. Simply find the largest negative $y_{i, j}$ and add the absolute value of this number to all the $y_{1, j}$ 's. Now all the $y_{i, j}$ 's are positive and use these to determine the $Z_{i, j}$ in the construction. Set the threshold $T$, attached to the sum of the final output, to this largest negative $y_{i, j}$ and it will rescale down the values of all the $y_{i, j}$ 's to the original data set.

The output of the network made from this construction has some distinct characteristics. Consider the case of non-negative $y_{i,}$ 's, which is equivalent to the case

\footnotetext{
'Note that we made the assumption in step 4 that $0<Z_{i, j}<1$. Also the determinations of the $T_{j}$ 's was independent of the $Z_{1, j}$ 's. So, whatever we do to the $Z_{i, j}$ 's in this step will not affect step 5.
} 
of allowing negative $y_{i, j}$ 's once rescaling is taken into account. Since the outputs of the nodes of the second layer are active only for a narrow region that includes each point that is interpolated, the function that the network computes will have either the value zero or $\gamma$ most of the time. Only around the narrow regions that include each point that is interpolated will the function not have one of these two values. Hence, this algorithm has no practical value, and is only of theoretical interest. However, it is still a polynomial time algorithm. 


\section{Chapter 5}

\section{Asymptotic Sigmoid Bounds}

In this chapter we prove that a two-layer network with asymptotic sigmoid artivation functions, satisfying certain conditions, having $k$ nodes on the first layer and $d$ nodes on the second layer, can interpolate any $k d$ points within an error of any arbitrarily small epsilon greater than zero. The structure of the network is the same as the $\pi$-network in the previous chapter, but it will have different activation functions. The proof is by construction. However, the considerations that must be taken into account are fundamentally different than those for the two-layer $\pi$-network. The sigmoids considered here never have constant values, which was the prime factor in the construction of the $\pi$-network. However, the idea behind the proof is essentially the same. Only one node on the second layer will be considered active, to within an epsilon, for each point to be interpolated. The first level of the network can not divide the $x$-axis into disjoint regions as in the construction of the $\pi$-network. As a result, new conceptual machinery must be devised for this construction. The main tool will be linear algebra sprinkled with some calculus. The proof will be presented by motivating the concepts that will be needed and the statements of the theorems will come at the end.

Let us consider a sigmoid network which has a real input and a real output. That is, it calculates the function $\theta-\operatorname{net}(x): \Re \rightarrow \Re$. If we just consider the $k$ outputs of the first layer, we can regard it as computing a function from $\Re \rightarrow \Re^{k}$. This is a path in $\Re^{k}$ space. Let $\theta_{i}$ be a function computed by the $i$-th first layer node of the 
network. The path $\rho$ that the first layer calculates is $\rho(x)=\left(\theta_{1}(x), \theta_{2}(x), \ldots, \theta_{k}(x)\right)$. A single node of the second layer with the outputs from the first layer as inputs would calculate the function $Z=\theta_{t}\left(W_{1} \theta_{1}(x)+W_{2} \theta_{1}(x)+\cdots+W_{k} \theta_{k}(x)-T\right)$. If we want this node to interpolate the $k$ values $z_{1}, z_{2}, \cdots, z_{k}$ in $[0,1]$ at $k$ different points as

$$
\begin{gathered}
\left(\left(y_{1,1}, y_{1,2}, \cdots, y_{1, k}\right), z_{1}\right) \\
\left(\left(y_{2,1}, y_{3,2}, \cdots, y_{2, k}\right), z_{2}\right) \\
\vdots \\
\left(\left(y_{k, 1}, y_{k, 2}, \cdots, y_{k, k}\right), z_{k}\right)
\end{gathered}
$$

we need

$$
\begin{array}{cc}
z_{1}= & \theta_{t}\left(W_{1} y_{1,1}+W_{2} y_{1,2}+\cdots+W_{k} y_{1, k}-T\right) \\
z_{1}= & \theta_{t}\left(W_{1} y_{2,1}+W_{2} y_{2,2}+\cdots+W_{k} y_{2, k}-T\right) \\
\vdots & \vdots \\
z_{1}= & \theta_{t}\left(W_{1} y_{k, 1}+W_{2} y_{k, 2}+\cdots+W_{k} y_{k, k}-T\right)
\end{array}
$$

or equivalently

$$
\begin{array}{ccc}
W_{1} y_{1,1}+W_{2} y_{1,2}+\cdots+W_{k} y_{1, k}=\theta_{t}^{-1}\left(z_{1}\right)+T= & b_{1} \\
W_{1} y_{2,1}+W_{2} y_{2,2}+\cdots+W_{k} y_{2, k} & =\theta_{t}^{-1}\left(z_{2}\right)+T= & b_{2} \\
\vdots & \vdots & \vdots \\
W_{1} y_{k, 1}+W_{2} y_{k, 2}+\cdots+W_{k} y_{k, k}= & \theta_{t}^{-1}\left(z_{k}\right)+T=b_{k}
\end{array}
$$

In matrix form this is expressed as

$$
\left[\begin{array}{cccc}
y_{1,1} & y_{1,2} & \cdots & y_{1, k} \\
y_{2,1} & y_{2,2} & \cdots & y_{2, k} \\
\vdots & \vdots & & \vdots \\
y_{k, 1} & y_{k, 2} & \cdots & y_{k, k}
\end{array}\right]\left[\begin{array}{c}
W_{1} \\
W_{2} \\
\vdots \\
W_{k}
\end{array}\right]=\left[\begin{array}{c}
b_{1} \\
b_{2} \\
\vdots \\
b_{k}
\end{array}\right]
$$

which we abbreviate as $Y u=b$.

A fundamental result of linear algebra is that these $k$ equations with arbitrary $b_{1}, b_{2}, \ldots, b_{k}$ will always have a solution for the variables $W_{1}, W_{2}, \ldots, W_{k}$ only if the $k$ rows of $r$ are linearly independent. A row is linearly independent if it can not be expressed as a linear combination of other rows. Thus if we want a node of the 
second level to be able to interpolate all $k$ of the above points we require that any $k$ points on the path $\rho(x)=\left(\theta_{1}(x), \theta_{2}(x) \ldots \theta_{k}(x)\right)$ be linearly independent. Stated below are some elementary lemmas about linear independence. presented without proofs. They will be used in the proofs of later lemmas.

LEMMA $5.1 n$ vectors $V_{1}^{\prime}, V_{2}, \ldots, V_{n}$, including points and functions, are linfarly independent if and only if $c_{1} V_{1}+c_{2} V_{2}+\cdots+c_{n} V_{n}=0$ has only the solution $c_{1}=$ $c_{2}=\cdots=c_{n}=0$.

LEMMA 5.2 The following conditions are equivalent for an $n \times n$ matrix A:

1. The rows of $A$ are linearly independent in $\Re^{n}$.

2. The rows of $A$ span $\Re^{n}$.

3. The columns of $A$ are linearly independent in $\Re^{n}$.

4. The columns of $A$ span $\Re^{n}$.

5. $A$ is invertible.

6. The determinant of $A$ is not equal to zero, i.e., $\operatorname{det} A \neq 0$.

Definition: $A$ linearly independent path $\rho: \Re \rightarrow \Re^{k}$ is a path such that any $k$ distinct points on the path are linearly independent.

Note that it is never possible for $k+1$ distinct points to be linearly independent since the number of linearly independent points can never be greater than the dimension of the space that they are contained in.

The following lemma provides the essential condition for a path to be linearly independent.

LEMMA 5.3 A path $\rho(x)=\left(\theta_{1}(x), \theta_{2}(x), \ldots, \theta_{k}(x)\right)$ is linearly independent if and only if the mapping coordinates $\theta_{1}(x)$ are linearly independent. 


\section{Proof}

Assume a linearly independent mapping. From lemma 5.1 we get that $c_{1} \theta_{1}(x)+$ $c_{2} \theta_{2}(x)+\cdots+c_{k} \theta_{k}(x)=0$ only has the solution $c_{1}=c_{2}=\cdots=c_{k}=0$. Substituting any $k$ arbitrary distinct points gives the following equations:

$$
\begin{aligned}
& c_{1} \theta_{1}\left(x_{1}\right)+c_{2} \theta_{2}\left(x_{1}\right)+\cdots+c_{k} \theta_{k}\left(x_{1}\right)=0 \\
& c_{1} \theta_{1}\left(x_{2}\right)+c_{2} \theta_{2}\left(x_{2}\right)+\cdots+c_{k} \theta_{k}\left(x_{2}\right)=0 \\
& c_{1} \theta_{1}\left(x_{k}\right)+c_{2} \theta_{2}\left(x_{k}\right)+\cdots+c_{k} \theta_{k}\left(x_{k}\right)=0
\end{aligned}
$$

In matrix form this is expressed as

$$
\left[\begin{array}{cccc}
\theta_{1}\left(x_{1}\right) & \theta_{2}\left(x_{1}\right) & \cdots & \theta_{k}\left(x_{1}\right) \\
\theta_{1}\left(x_{2}\right) & \theta_{2}\left(x_{2}\right) & \cdots & \theta_{k}\left(x_{2}\right) \\
\vdots & \vdots & & \vdots \\
\theta_{1}\left(x_{k}\right) & \theta_{2}\left(x_{k}\right) & \cdots & \theta_{k}\left(x_{k}\right)
\end{array}\right]\left[\begin{array}{c}
c_{1} \\
c_{2} \\
\vdots \\
c_{k}
\end{array}\right]=\left[\begin{array}{c}
0 \\
0 \\
\vdots \\
0
\end{array}\right]
$$

Now assume a linearly independent path. Using the same arbitrary points used in (5.3), from lemma 5.1 we get that

$$
\begin{aligned}
c_{1}\left(\theta_{1}\left(x_{1}\right), \theta_{2}\left(x_{1}\right), \ldots, \theta_{k}\left(x_{1}\right)\right)+ & c_{2}\left(\theta_{1}\left(x_{2}\right), \theta_{2}\left(x_{2}\right), \ldots, \theta_{k}\left(x_{2}\right)\right)+\cdots \\
& +c_{k}\left(\theta_{1}\left(x_{k}\right), \theta_{2}\left(x_{k}\right), \ldots, \theta_{k}\left(x_{k}\right)\right)=0
\end{aligned}
$$

has only the solution $c_{1}=c_{2}=\cdots=c_{k}=0$. Collecting according to dimension gives

$$
\begin{array}{ccc}
c_{1} \theta_{1}\left(x_{1}\right)+c_{2} \theta_{1}\left(x_{2}\right)+\cdots+c_{k} \theta_{1}\left(x_{k}\right)= & 0 \\
c_{1} \theta_{2}\left(x_{1}\right)+c_{2} \theta_{2}\left(x_{2}\right)+\cdots+c_{k} \theta_{2}\left(x_{k}\right)= & 0 \\
\vdots & \vdots & \vdots \\
c_{1} \theta_{k}\left(x_{1}\right)+c_{2} \theta_{k}\left(x_{2}\right)+\cdots+c_{k} \theta_{k}\left(x_{k}\right)= & 0
\end{array}
$$

In matrix form this is expressed as

$$
\left[\begin{array}{cccc}
\theta_{1}\left(x_{1}\right) & \theta_{1}\left(x_{2}\right) & \cdots & \theta_{1}\left(x_{k}\right) \\
\theta_{2}\left(x_{1}\right) & \theta_{2}\left(x_{2}\right) & \cdots & \theta_{2}\left(x_{k}\right) \\
\vdots & \vdots & & \vdots \\
\theta_{k}\left(x_{1}\right) & \theta_{k}\left(x_{1}\right) & \cdots & \theta_{k}\left(x_{1}\right)
\end{array}\right]\left[\begin{array}{c}
c_{1} \\
c_{2} \\
\vdots \\
c_{k}
\end{array}\right]=\left[\begin{array}{c}
0 \\
0 \\
\vdots \\
0
\end{array}\right]
$$


Notice that the columns of (5.4) are the same as the rows of (5.5) and vice versa. From lemma 5.2 it follows that if the rows of a matrix are linearly independent then the columns of the matrix are also linearly independent. Thus the linear independence of (5.4) implies the linear independence of (5.5) and vice versa.

The following lemma gives a sufficient condition for a path $\rho(x)$ to be linearly. independent.

LEMMA $5.4 A$ sufficient condition for a path $\rho(x)=\left(\theta_{1}(x), \theta_{2}(x), \ldots, \theta_{k}(x)\right)$ to br linearly independent is

$$
\operatorname{det}\left[\begin{array}{cccc}
\theta_{1}^{(1)}(x) & \theta_{2}^{(1)}(x) & \cdots & \theta_{k}^{(1)}(x) \\
\theta_{1}^{(2)}(x) & \theta_{2}^{(2)}(x) & \cdots & \theta_{k}^{(2)}(x) \\
\vdots & \vdots & & \vdots \\
\theta_{1}^{(k)}(x) & \theta_{2}^{(k)}(x) & \cdots & \theta_{k}^{(k)}(x)
\end{array}\right] \neq 0 \text {, for all } x \in \Re,
$$

where $\theta_{i}^{(k)}(x)$ refers to the $k$-th derivative of $\theta_{1}(x)$ with respect to $x$.

\section{Proof}

We must show that the above condition implies that the only solution of

$$
c_{1} \theta_{1}(x)+c_{2} \theta_{2}(x)+\cdots+c_{k} \theta_{k}(x)=0 \text { is } c_{1}=c_{2}=\cdots=c_{k}=0 .
$$

Taking the first $\mathrm{k}$ derivatives of $c_{1} \theta_{1}(x)+c_{2} \theta_{2}(x)+\cdots+c_{k} \theta_{k}(x)=0$ gives

$$
\begin{aligned}
& c_{1} \theta_{1}^{(1)}(x)+c_{2} \theta_{2}^{(1)}(x)+\cdots+c_{k} \theta_{k}^{(1)}(x)=0 \\
& c_{1} \theta_{1}^{(2)}(x)+c_{2} \theta_{2}^{(2)}(x)+\cdots+c_{k} \theta_{k}^{(2)}(x)=0 \\
& c_{1} \theta_{1}^{(k)}(x)+c_{2} \theta_{2}^{(k)}(x)+\cdots+c_{k} \theta_{k}^{(k)}(x)=0
\end{aligned}
$$

Equivalently,

$$
\left[\begin{array}{cccc}
\theta_{1}^{(1)}(x) & \theta_{2}^{(1)}(x) & \cdots & \theta_{k}^{(1)}(x) \\
\theta_{1}^{(2)}(x) & \theta_{2}^{(2)}(x) & \cdots & \theta_{k}^{(2)}(x) \\
\vdots & \vdots & & \vdots \\
\theta_{1}^{(k)}(x) & \theta_{2}^{(k)}(x) & \cdots & \theta_{k}^{(k)}(x)
\end{array}\right]\left[\begin{array}{c}
c_{1} \\
c_{2} \\
\vdots \\
c_{k}
\end{array}\right]=\left[\begin{array}{c}
0 \\
0 \\
\vdots \\
0
\end{array}\right]
$$


lirom lemma 5.2, the non zero determinant of the left-most matrix implies that it is linearly independent. It follows from lemma 5.1 that the only solution of $(5.6)$ is when $c_{1}=c_{2}=\cdots=c_{2}=0$.

We have now proved that (5.2), $Y w=b$ always has a solution $w$, the weight matrix, for arbitrary $b$, where $Y$ represents any $k$ points generated fron a linearly independent path in $k$-space. This solution may be found by simple Gauss-Jordan elimination. The solution of $w$ represents the assignment of weights to a single node on the second layer of the network. However, hidden in column $b$ is the threshold $T$, which can $t$. set to anything before $w$ is solved.

Notice that the above theorem is very general. No assumptions have yet been made about any of the node functions being sigmoidal. The only assumptions made so far are that the functions of the first layer, that define the path $\rho$, satisfy the sufficiency conditions of lemma 5.4 and the node $\theta_{t}(x)$ of the second level is invertible and continuous. It is not required in our construction that the nodes of the first layer be sigmoidal. However, whatever sigmoids that are used must be shown to satisfy the sufficiency condition. Only the nodes of the second layer are required to be sigmoids. This will follow from the nature of our construction in which we do not want the nodes of the second layer to "interact". The asymptotic properties of sigmoids will be used to achieve this.

The value of $T$ will be assigned in such a way as to ensure that for each point interpolated by a node in the second layer of the network, the outputs of the other nodes of the second layer can be regarded as constants for the same points.

Unlike the $\pi$-activation function, the outputs of sigmoids that only reach their limiting values asymptotically are never true constant values. However, they can be regarded as constants within a certain value.

Definition: The $\delta$-astivation region of a sigmoid $\theta_{t}(x)$ is the region of $x$ where $\delta<\theta_{t}(x)<1-\delta$. Outside of this region the value of $\theta_{t}(x)$ can be regarded as a constant, 0 or 1 , within a value of $\delta$. 
If $x=\left(x_{1}, x_{2}, \cdots, x_{k}\right) \in \Re^{k}$ then for $\theta_{t}\left(W_{1} x_{1}+W_{2} x_{2}+\cdots+W_{k} x_{k}-T\right)$ the $\delta$-activation region is the region enclosed between the hyperplanes

$$
\begin{aligned}
& W_{1} x_{1}+W_{2} x_{2}+\cdots+W_{k} x_{k}-T-\theta_{t}^{-1}(\delta)=0, \text { and } \\
& W_{1} x_{1}+W_{2} x_{2}+\cdots+W_{k} x_{k}-T-\theta_{t}^{-1}(1-\delta)=0 .
\end{aligned}
$$

Notice that they are parallel. The width. $\Delta_{\delta}$, of the $\delta$-activation region is the perpendicular distance between the two surfaces of the hyperplanes. This is equal to

$$
\Delta_{\delta}=\frac{\theta_{t}^{-1}(1-\delta)-\theta_{t}^{-1}(\delta)}{\sqrt{W_{1}^{2}+W_{2}^{2}+\cdots+W_{k}^{2}}}
$$

Therefore,

$$
W_{i} \leq \frac{\theta_{\ell}^{-1}(1-\delta)-\theta_{i}^{-1}(\delta)}{\Delta_{\delta}}, \text { for } 1 \leq i \leq k .
$$

Thus, the width of the activation region shrinks as the $W_{i}$ 's increases. To find the relationship between the width of the activation region and the threshold $\mathrm{T}$, refer back to (5.2)

$$
\left[\begin{array}{cccc}
y_{1,1} & y_{1,2} & \cdots & y_{1, k} \\
y_{2,1} & y_{2,2} & \cdots & y_{2, k} \\
\vdots & \vdots & & \vdots \\
y_{k, 1} & y_{k, 2} & \cdots & y_{k, k}
\end{array}\right]\left[\begin{array}{c}
W_{1} \\
W_{2} \\
\vdots \\
W_{k}
\end{array}\right]=\left[\begin{array}{c}
\theta_{t}^{-1}\left(z_{1}\right)+T \\
\theta_{\imath}^{-1}\left(z_{2}\right)+T \\
\vdots \\
\theta_{\imath}^{-1}\left(z_{k}\right)+T
\end{array}\right]
$$

Using Cramers's rule to solve for $W_{1}$ gives

$$
W_{1}=\frac{\operatorname{det}\left[\begin{array}{cccc}
\theta_{t}^{-1}\left(z_{1}\right)+T & y_{1,2} & \cdots & y_{1, k} \\
\theta_{t}^{-1}\left(z_{2}\right)+T & y_{2,2} & \cdots & y_{2, k} \\
\vdots & \vdots & & \vdots \\
\theta_{t}^{-1}\left(z_{2}\right)+T & y_{k, 2} & \cdots & y_{k, k}
\end{array}\right]}{\operatorname{det}\left[\begin{array}{cccc}
y_{1,1} & y_{1,2} & \cdots & y_{1, k} \\
y_{2,1} & y_{2,2} & \cdots & y_{2, k} \\
\vdots & \vdots & & \vdots \\
y_{k, 1} & y_{k, 2} & \cdots & y_{k, k}
\end{array}\right]}
$$




$$
=\frac{\operatorname{Tdet}\left[\begin{array}{cccc}
1 & y_{1,2} & \cdots & y_{1, k} \\
1 & y_{2,2} & \cdots & y_{2, k} \\
\vdots & \vdots & & \vdots \\
1 & y_{k, 2} & \cdots & y_{k, k}
\end{array}\right]+\operatorname{det}\left[\begin{array}{ccccc}
\theta_{t}^{-1}\left(z_{1}\right) & y_{1,2} & \cdots & y_{1, k} \\
\theta_{t}^{-1}\left(z_{2}\right) & y_{2,2} & \cdots & y_{2, k} \\
\vdots & \vdots & & \vdots \\
\theta_{t}^{-1}\left(z_{2}\right) & y_{k, 2} & \cdots & y_{k, k}
\end{array}\right]}{\operatorname{det}\left[\begin{array}{cccc}
y_{1,1} & y_{1,2} & \cdots & y_{1, k} \\
y_{2,1} & y_{2,2} & \cdots & y_{2, k} \\
\vdots & \vdots & & \cdot \\
y_{k, 1} & y_{k, 2} & \cdots & y_{k, k}
\end{array}\right]}
$$

$$
\begin{gathered}
\text { Since } W_{1} \leq \frac{\theta_{t}^{-1}(1-\delta)-\theta_{t}^{-1}(\delta)}{\Delta_{\delta}} \text { we get } \\
\Delta_{\delta} \leq \frac{\left(\theta_{t}^{-1}(1-\delta)-\theta_{t}^{-1}(\delta)\right) \operatorname{det}\left[\begin{array}{cccc}
y_{1,1} & y_{1,2} & \cdots & y_{1, k} \\
y_{2,1} & y_{2,2} & \cdots & y_{2, k} \\
\vdots & \vdots & & \vdots \\
y_{k, 1} & y_{k, 2} & \cdots & y_{k, k}
\end{array}\right]}{T \operatorname{det}\left[\begin{array}{cccc}
1 & y_{1,2} & \cdots & y_{1, k} \\
1 & y_{2,2} & \cdots & y_{2, k} \\
\vdots & \vdots & & \vdots \\
1 & y_{k, 2} & \cdots & y_{k, k}
\end{array}\right]+\operatorname{det}\left[\begin{array}{cccc}
\theta_{t}^{-1}\left(z_{1}\right) & y_{1,2} & \cdots & y_{1, k} \\
\theta_{t}^{-1}\left(z_{2}\right) & y_{2,2} & \cdots & y_{2, k} \\
\vdots & \vdots & & \vdots \\
\theta_{t}^{-1}\left(z_{2}\right) & y_{k, 2} & \cdots & y_{k, k}
\end{array}\right]}
\end{gathered}
$$

Thus we can determine an upper bound on the width of the? activation region in terms of $T$ only. It is clear that as $T$ increases the width decreases and $\Delta_{6}$ can be made arbitrarily small by making $T$ as large as required.

A point $x_{0}$ being interpolated by a node on the second level lies on the hyperplane $W_{0} \theta_{1}\left(x_{0}\right)+W_{1} \theta_{1}\left(x_{0}\right)+\cdots+W_{k} \theta_{k}\left(x_{0}\right)-\theta_{j}^{-1}\left(z_{0}\right)-T=0$, which is in the $\delta$-activation region. It is required that any point $x_{0}+\epsilon, \epsilon>0$ which is arbitrarily close to $x_{0}$ be outside the $\delta$-activation region. Since the width of the $\delta$-activation region can be made arbitrarily small by making $T$ arbitrarily large and the boundaries of the $\delta$-activation region are parallel to the hyperplane that interpolates point $x_{0}$, it is 
enough to show that the hyperplane at a point $x_{0}+\epsilon, c>0$, parallel to the one that interpolates point $x_{0}$, are not identical. If there existed parallel hyperplanes at points $x_{0}$ and $x_{0}+\epsilon$ that were identical then the two points would lie in the $\delta$-activation region, which is not desired for our construction.

LEMMA 5.5 Given a path $\rho(x)=\left(\theta_{1}(x), \theta_{2}(x), \ldots, \theta_{k}(x)\right)$. A necessary condition for the existence of an $\epsilon>0$ such that no points in the regions $\left(\rho\left(x_{0}-\epsilon\right), \rho\left(x_{0}\right)\right)$ and $\left(\rho\left(x_{0}\right), \rho\left(x_{0}+\epsilon\right)\right)$ lie on the same $k-1$ dimensional hyperplane passing through an arbitrary point $\rho\left(x_{0}\right)$ in $k$-space is

$$
\operatorname{det}\left[\begin{array}{cccc}
\theta_{1}^{(1)}(x) & \theta_{2}^{(1)}(x) & \cdots & \theta_{k}^{(1)}(x) \\
\theta_{1}^{(2)}(x) & \theta_{2}^{(2)}(x) & \cdots & \theta_{k}^{(2)}(x) \\
\vdots & \vdots & & \vdots \\
\theta_{1}^{(k)}(x) & \theta_{2}^{(k)}(x) & \cdots & \theta_{k}^{(k)}(x)
\end{array}\right] \neq 0 \text {, for all } x \in \Re .
$$

\section{Proof}

The proof will be by showing that the points $\rho\left(x_{0}\right)$ and $\rho\left(x_{0}+\epsilon\right)$ could lie on the same hyperplane only if the above matrix had a zero determinant. The case of $\rho\left(x_{0}-\epsilon\right)$ is nearly identical to that of $\rho\left(x_{0}+\epsilon\right)$. Just replace $\epsilon$ by $-\epsilon$ in the proof below.

Assume that the points $\rho\left(x_{0}\right)$ and $\rho\left(x_{0}+\epsilon\right), \epsilon>0$ lie on the same hyperplane. This gives

$$
\begin{aligned}
& d=W_{1} \theta_{1}\left(x_{0}\right)+W_{2} \theta_{2}\left(x_{0}\right)+\cdots+W_{k} \theta_{k}\left(x_{0}\right), \\
& d=W_{1} \theta_{1}\left(x_{0}+\epsilon\right)+W_{2} \theta_{2}\left(x_{0}+\epsilon\right)+\cdots+W_{k} \theta_{k}\left(x_{0}+\epsilon\right) .
\end{aligned}
$$

We use the definition of the $i$-th derivative,

$$
\lim _{\epsilon \rightarrow 0} \frac{\theta_{j}^{(i-1)}\left(x_{0}+\epsilon\right)-\theta_{j}^{(i-1)}\left(x_{0}\right)}{\epsilon}=\theta_{j}^{(i)}\left(x_{0}\right)
$$

or equivalently

$$
\theta_{j}^{(i-1)}\left(x_{0}+\epsilon\right)=\epsilon \theta_{j}^{(1)}\left(x_{0}\right)+\theta_{j}^{(i-1)}\left(x_{0}\right)+o(\epsilon), \text { where } \lim _{\epsilon \rightarrow 0} \frac{o(\epsilon)}{\epsilon}=0 .
$$


Expanding (5.9) using (5.10) with $i=1$ gives

$$
\begin{aligned}
d=W_{1}\left[\epsilon \theta_{1}^{(1)}\left(x_{0}\right)+\theta_{1}\left(x_{0}\right)+o(\epsilon)\right]+W_{2} & {\left[\epsilon \theta_{2}^{(1)}\left(x_{0}\right)+\theta_{2}\left(x_{0}\right)+o(\epsilon)\right]+\cdots } \\
& +W_{k}\left[\epsilon \theta_{k}^{(1)}\left(x_{0}\right)+\theta_{k}\left(x_{0}\right)+o(\epsilon)\right]
\end{aligned}
$$

Note that $W_{1} o(\epsilon)+W_{2} o(\epsilon)+\cdots+W_{k} o(\epsilon)=o(\epsilon)$. Subtracting (5.8) from (5.11) gives

$$
o(\epsilon)=W_{1} \epsilon \theta_{1}^{(1)}\left(x_{0}\right)+W_{2} \epsilon \theta_{2}^{(1)}\left(x_{0}\right)+\cdots+W_{k} \epsilon \theta_{k}^{(1)}\left(x_{0}\right) .
$$

Assuming $\epsilon \neq 0$,

$$
\frac{o(\epsilon)}{\epsilon}=W_{1} \theta_{1}^{(1)}\left(x_{0}\right)+W_{2} \theta_{2}^{(1)}\left(x_{0}\right)+\cdots+W_{k} \theta_{k}^{(1)}\left(x_{0}\right) .
$$

Similarly $k-1$ more equations can be found by taking the $i$-th derivative of (5.9) expanding it with (5.10) and subtracting the $i$-th derivative of (5.8), for $1 \leq i \leq k-1$. The resulting system of equations is

$$
\begin{gathered}
{\left[\begin{array}{cccc}
\theta_{1}^{(1)}\left(x_{0}\right) & \theta_{2}^{(1)}\left(x_{0}\right) & \cdots & \theta_{k}^{(1)}\left(x_{0}\right) \\
\theta_{1}^{(2)}\left(x_{0}\right) & \theta_{2}^{(2)}\left(x_{0}\right) & \cdots & \theta_{k}^{(2)}\left(x_{0}\right) \\
\vdots & \vdots & & \vdots \\
\theta_{1}^{(k)}\left(x_{0}\right) & \theta_{2}^{(k)}\left(x_{0}\right) & \cdots & \theta_{k}^{(k)}\left(x_{0}\right)
\end{array}\right]\left[\begin{array}{c}
W_{1} \\
W_{2} \\
\vdots \\
W_{k}
\end{array}\right]=\left[\begin{array}{c}
\frac{o(c)}{e} \\
\frac{o(c)}{e} \\
\vdots \\
\frac{o(c)}{c}
\end{array}\right]} \\
\text { In the limit as } \epsilon \rightarrow 0,\left[\begin{array}{c}
\frac{o(c)}{e} \\
\frac{o(e)}{c} \\
\vdots \\
\frac{o(c)}{e}
\end{array}\right]=\left[\begin{array}{c}
0 \\
0 \\
\vdots \\
0
\end{array}\right]
\end{gathered}
$$

In the limit as $\epsilon \rightarrow 0$, for the $W$ matrix in (5.13) to have a non-trivial solution the left-most matrix must be linearly dependent by lemma 5.1 or equivalently have a zero determinant.

It follows that a single node of the second layer with $k$ inputs from a special linearly independent path can interpolate any $k$ points with an output value in $[0,1]$. Now that we have finished defining, motivating, and proving the fundamental concepts, we state in one theorem all that has been proved up to now. 
Theorem 5.1 Let $\theta_{t}, 1<t<k$, be continuous sigmoids with $\lim _{s--\infty} \theta_{t}(x)=$ 0 and $\lim _{x \rightarrow \infty} \theta_{t}(x)=1$ and let path $\rho(x)=\left(\theta_{1}(x), \theta_{2}(x) \ldots, \theta_{k}(x)\right)$. Assume $k$ distinct points $\left(\left(x_{1}, z_{1}\right),\left(x_{2}, z_{2}\right), \ldots,\left(x_{k}, z_{k}\right)\right), x_{1} \in \Re, z_{1} \in[0,1], 1<1<k . A$ necessary and sufficient condition for there to exist $w_{1}, w_{2}, \ldots, w_{k}$ and $T \in \Re$ such that $z_{1}=\theta_{t}\left(W_{1} \theta_{1}\left(x_{1}\right)+W_{2} \theta_{1}\left(x_{1}\right)+\cdots+W_{k} \theta_{k}\left(x_{1}\right)-T\right), 1<i<k$, and outside an arbitrarily small region that includes each point the output may be rigarded as a constant, 0 or 1 , within an arbitrary $\delta$ is

$$
\operatorname{det}\left[\begin{array}{cccc}
\theta_{1}^{(1)}(x) & \theta_{2}^{(1)}(x) & \cdots & \theta_{k}^{(1)}(x) \\
\theta_{1}^{(2)}(x) & \theta_{2}^{(2)}(x) & \cdots & \theta_{k}^{(2)}(x) \\
\vdots & \vdots & & \vdots \\
\theta_{1}^{(k)}(x) & \theta_{2}^{(k)}(x) & \cdots & \theta_{k}^{(k)}(x)
\end{array}\right] \neq 0 \text {, for all } x \in \Re .
$$

Intuitively, what is happening is that the path intersects a thin $k-1$ dimensional hyperplane, that represents the $\delta$-activation region, in $k$-space at each of the $k$ points. The $k$-space represents the output values of a single node under all possible inputs. The path represents the outputs of the nodes on the first layer. The values in $k$-space aing the path represent the output of a node on the second level under all possible inputs to the first layer. The region in $k$-space above the $k-1$ dimensional hyperplane can be regarded as having the value one and below as having the value zero. Each point on the path (all points, not only the ones being interpolated) can only be one of above, below, or in the $\delta$-activation region. If the value of a segment of the path to the immediate left of a point in the $\delta$-activation region can be regarded as a one then the value of the segment of the path to the immediate right of that point must be regarded as a zero. This is because the portion of the path, including the point and both segments, has just passed through the $\delta$-activation region. Thus, every time a segment of the path passes through $\delta$-activation region the value of the adjacent segment on the other side must change from a constant, 0 or 1 , to the other constant.

Consider a node of the second layer interpolating $k$ points. That is, the path passes through the activation region at the appropriate points $k$ times for their appropriate values. To the left of the left-most point on the real number line, the 
output will be one of the constants. As the path passes through the $\delta$-activation region $k$ times the constant value will change $k$ times. Thus, to determine the output to the right of the right-most point, $k$ constant value changes must be taken into consideration. If $k$ is even, then the output to the right of all the points will have the same constant output as to the left of all the points. However, if $k$ is odd it will have the opposite constant value because the path has passed through the $\delta$-activation region an odd number of times.

For the sake of convenience, the output to the left of all the points that a node on the second layer interpolates can be regarded as zero. This can always be done by reversing the orientation of the hyperplanes that represent the $\delta$-activation region. All that needs to be done is to change the sign of the threshold $T$ and resolve the equations (5.2). For large $T$ it is evident from Cramer's rule, see (5.7), that the sign of the weights will also change. Thus the path $\rho(x)$ can be remapped to $-\rho(x)$ for that node.

Now that we have taken care of the constant values for the output of a single node of the second layer, we can figure out how to combine the outputs of all $d$ nodes of the second layer to interpolate any $k d$ points. The $k d$ points will be divided into $d$ regions with $k$ points in each of the regions. The $j$-th node will interpolate the $k$ points in the $j$-th region.

Note that this is the opposite strategy as used in the construction of the two layer $\pi$-network, where $k d$ puints were divided into $k$ regions with $d$ points in each region. Each node of the the second layer interpolated one point from each of the $k$ regions. The same strategy could be used here. However, the construction would not be as easy to explain. The strategy employed here could not be used for the $\pi$-network because of the linear nature of its activation regions.

The $k d$ points to be interpolated are labeled as

$$
p_{i, j}=\left(x_{i, j}, y_{i, j}\right): 1 \leq i \leq k, 1 \leq j \leq d,
$$

with

$$
x_{1,1}<x_{1,2}<\cdots<x_{1, k}<x_{2,1}<x_{2,2}<\cdots<x_{2, k}<\cdots<x_{d, 1}<x_{d, 2}<\cdots<x_{d, k} .
$$


For the moment consider all the $y_{i, j}$ 's as positive.

There are two cases to consider when constructing the network; one when $k$ is even and the other when $k$ is odd. When $k$ is even, simply set all the weights of the outputs from the second layer to the maximum value of the $y_{0, j} s$. Call this $q$. The $z_{h}$ 's that are to be interpolated for each node $g$ by $(5.1)$ are set as

$$
z_{h}=\frac{y_{g . h}}{\gamma}, 1 \leq h \leq k .
$$

The weights can be set this way because the outputs for each node of the second layer outside its region of assigned points will be zero with certainty $\delta$. Thus the certainty of the final value interpolated is $(d-1) \delta$, which can be regarded as an $\epsilon$. Thus this certainty can be made arbitrarily small.

For the case of odd $k$, the same strategy is used as for the two-layer $\pi$-network; alternately assigning to the weights the values $\gamma$ and $-\gamma$. Once again the uncertainty is $(d-1) \delta$, which can be regarded as an $\epsilon$.

If there are negative $y_{i,}$ 's then the same rescaling method is used as in the construction of the two-layer $\pi$-network.

The main theorem, which we have proved, is stated.

Theorem 5.2 Let $\theta$ be a continuous sigmoid with $\lim _{x \rightarrow-\infty} \theta(x)=0$ and $\lim _{x \rightarrow \infty} \theta(x)=1$. If there exists $W_{i}^{\prime}$ and $T_{i}^{\prime}, 1 \leq i \leq k$ such that

$$
\operatorname{det}\left[\begin{array}{cccc}
\theta^{(1)}\left(W_{1}^{\prime} x-T_{1}^{\prime}\right) & \theta^{(1)}\left(W_{2}^{\prime} x-T_{2}^{\prime}\right) & \cdots & \theta^{(1)}\left(W_{k}^{\prime} x-T_{k}^{\prime}\right) \\
\theta^{(2)}\left(W_{1}^{\prime} x-T_{1}^{\prime}\right) & \theta^{(2)}\left(W_{2}^{\prime} x-T_{2}^{\prime}\right) & \cdots & \theta^{(2)}\left(W_{k}^{\prime} x-T_{k}^{\prime}\right) \\
\vdots & \vdots & & \vdots \\
\theta^{(k)}\left(W_{1}^{\prime} x-T_{1}^{\prime}\right) & \theta^{(k)}\left(W_{2}^{\prime} x-T_{2}^{\prime}\right) & \cdots & \theta^{(k)}\left(W_{k}^{\prime} x-T_{k}^{\prime}\right)
\end{array}\right] \neq 0, \text { for all } x \in \Re,
$$

then a two-layer network

$$
\theta-\operatorname{net}(x)=\sum_{j=1}^{d}\left[W_{j} \theta\left(\sum_{i=1}^{k}\left[W_{i, j}^{\prime \prime} \theta\left(W_{i}^{\prime} x-T_{i}^{\prime}\right)\right]-T_{j}^{\prime \prime}\right)\right]-T
$$

can interpolate any $k d$ points within any $\epsilon, \epsilon>0$, with the appropriate assignments to the weights and thresholds. 
Theorem 5.3 A two-layer network with the above architecture and the standard sigmoid $\theta$, as its activation function can interpolate any $k d$ points within any $\epsilon, \epsilon>0$.

\section{PrOOF}

Since $\theta_{\text {a }}$ is continuous we only need to show the existence of a non-zero determinant. We have $\theta_{s}^{\prime}(x)=\left(1-\theta_{s}(x)\right) \theta_{s}(x)$.

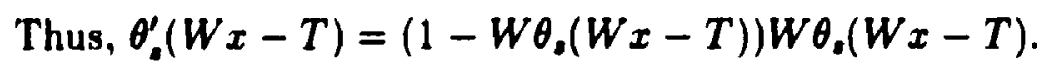

It is clear that $\theta_{a}^{(n)}(W x-T)$ will be of order $\left(\theta_{0}(W x-T)\right)^{n}$ Thus, the derivatives are linearly independent since $\theta_{3}$ is a strictly increasing function and polynomials of the first $n$ order are linearly independent. Now we only need to show that no two columns are ever the same for any $x$. Since $\theta$, is a strictly increasing function all that needs to be shown is that they never agree for any particular $W x-T$. This will be the case if

$$
\frac{T_{i}}{W_{i}} \neq \frac{T_{j}}{W_{j}}, i \neq j
$$

To help understand the nature of this two-layer construction see figure 5.1, which has two nodes on the first layer and two nodes on the second layer. The various graphs show the outputs of the nodes at the various levels. The bottom laver sigmoids $\theta_{1}(x)$ and $\theta_{2}(x)$ produce the linearly independent path. When they are graphed against each other they produce a curve, as can be seen in the shaded boxes of figure 5.1. This curve has the special property, in the two dimensional case, that it can be intersected only at most two distinct points by a one dimensiunal hyperplane (a line). The width of this line, on the diagram, represents the $\delta$-activation region. The light shading represents the region of 2-space where the activation has constant value zero and the dark shading represents the region where it has constant value one. As with the two-layer $\pi$-net the function it computes is thearly always one of the two constant values, except at the points being interpolated. 

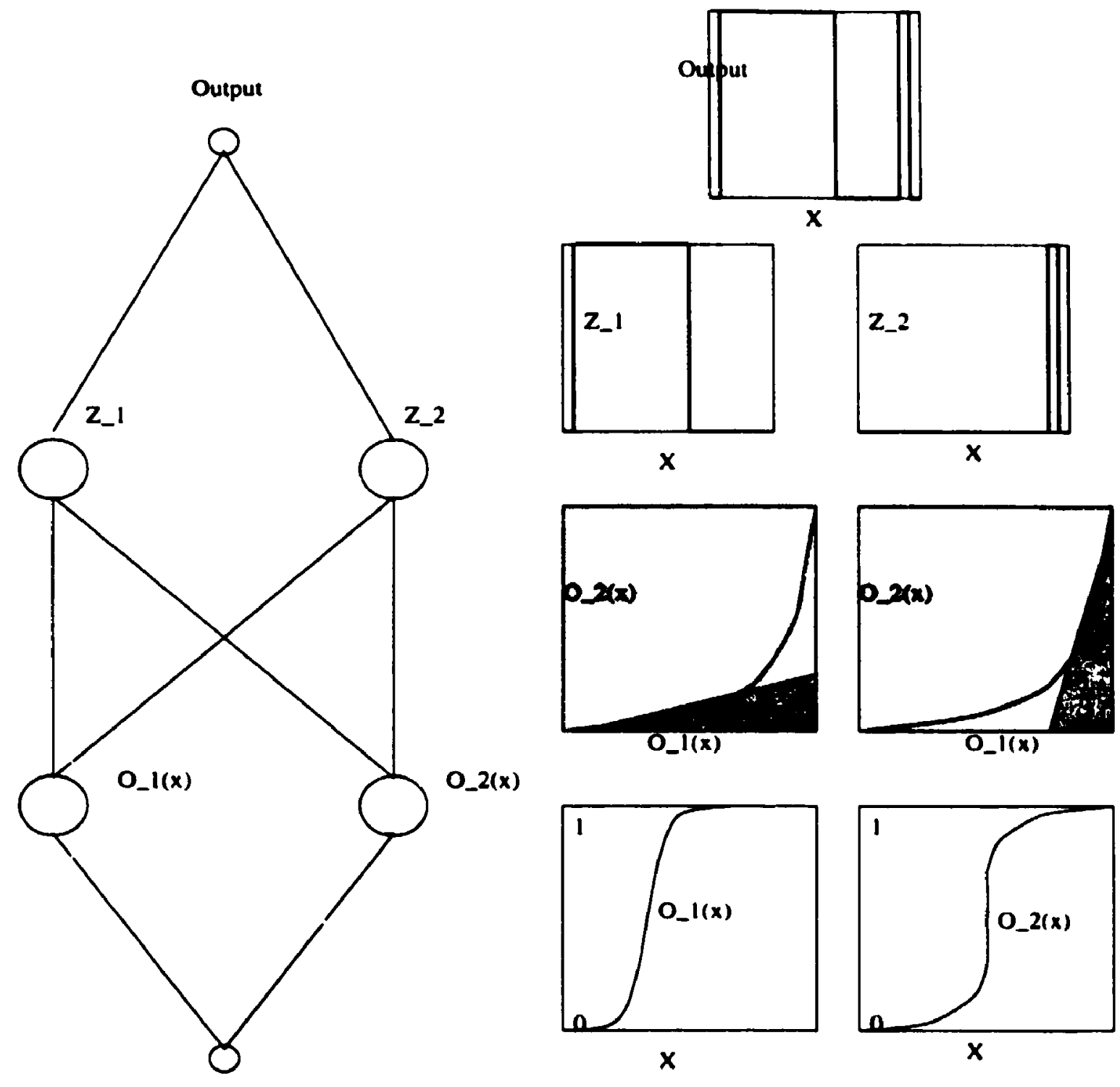

Figure 5.1: Outputs of a 2-2 network 


\section{Chapter 6}

\section{Conclusion}

Neural networks provide an exciting model of analog computation. Bounds on the interpolation capacity of neural networks are important to their theory because of the computational difficulty of training them. The best bounds currently known to date for one and two layer networks for the following activation functions are as follows.

\begin{tabular}{|c|c|c|c|}
\hline $\begin{array}{l}\text { Activation } \\
\text { Function }\end{array}$ & $\begin{array}{c}\text { Number of } \\
\text { layers }\end{array}$ & $\begin{array}{l}\text { Lower } \\
\text { bound }\end{array}$ & $\begin{array}{l}\text { Upper } \\
\text { bound }\end{array}$ \\
\hline$\pi$ & 1 & $2 k$ & $2 k$ \\
\hline$\pi$ & 2 & $\mathbf{k d}$ & unknown \\
\hline$\theta_{s}$ & 1 & $2 k-1$ & $2 k$ \\
\hline$\theta$ & 2 & kd & $k d+2 k+2 d$ \\
\hline
\end{tabular}

where for the multilayer cases, $k$ is the number of nodes on the first layer and $d$ is the number of nodes on the second layer.

The $2 k$ upper bound for the one-layer $\theta_{s}$ network and the two lower bounds for the tuo-layer networks were proved in this thesis. The lower bounds for the twolayer $\theta$, network was actually part of a more general theorem that is valid for a large class of asymptotic sigmoids.

The constructions for the lower bounds have no practical value as training algorithms since the functions the networks produce are almost always one of two 
constant values except for narrow regions that include the actual points being interpolated.

\section{Future Research}

Determining the precise values of the interpolation capacity of the various classes of networks found in this thesis is an interesting and challenging problem. The problem of finding bounds for the interpolation capacity of networks of arbitrary depth is also interesting. We conjecture that the lower bounds for a $\theta_{s}$-net with depth $h$ and having $k$ nodes on each layer is no less than $(h-1) k^{2}-(h-2) k$ and the lower bounds for a $\pi$-net with depth $h$ and having $k$ nodes on each layer is no less than $(k+h-2)(k+1)$. 


\section{Bibliography}

[1] J.A. Anderson and E. Rosenfeld, editors. Neurocomputing: Foundations of Research. MIT Press, Cambridge, 1988.

[2] A. Blum and R.L. Rivest. Training a 3-node neural net is np-complete. In D.S.

- Touretzky, editor, Advances in Neural Information Processing Systems I, pages 494-501. Morgan Kaufmann, San Mateo, CA, 1989.

[3] J. Boyan. Modular neural networks for learning context-dependent game strata. gies. Master's thesis, University of Cambridge, 1992. Department of Engineering and Computer Laboratory.

[4] G. Cybenko. Continuous valued neural networks with two hidden layers are sufficient. Technical report, Department of Computer Science, Tufts University, Medford, MA, 1988.

[5] G. Cybenko. Approximation by superpositions of a sigmoidal function. Mathematics of Control, Signals, and Systems, 2:303-314, 1989.

[6] S.E. Fahlman and C. Lebiere. The cascade-correlation learning architecture. In D.S. Tou.etzky, editor, Advances in Neural Information Processing Systems, volume 2, pages 524-532. Morgan Kaufmann, San Mateo, 1990.

[7] $k$. Funahashi. On the approximate realization of continuous mappings by neural networks. Neural Networks, 2:183-192, 1989.

[8] M.R. Garey and D.S. Johnson. Computers and Intractability: A Guide to the Theory of NP.Completeness. Freeman, New York, 1979. 
[9] K.-U. Höffgen. Computational limitations of training sigmoid neural networks. Technical Report 413, University of Dortmund. 1992.

[10] K.-C. Höffgen. Computational limitations on training sigmoid neural networks. In I. Aleksander and J. Taylor, editors, Artificial Neural Netuorks, 2., volume I, pages 109-112. Elsevier Science Publishing Company B. V., Ansterdam, 1992.

[11] K. Hornik. Approximation capabilities of multilayer feedforward networks. Neural Networks, 4:251-257, 1991.

[12] K. Hornik, M. Stinchcombe, and H. White. Multilayer feedforward networks are universal approximators. Neural Networks, 2:359-366, 1989.

[13] J.S. Judd. Neural Network Design and the Complexity of Learning. MIT Press, Cambridge, MA, 1990.

[14] Jones L. K. Constructive approximations for neural networks by sigmoidal functions. Proceedings of the IEEE, 78(10):1586-1589, 1990.

[15] Mangasarian O. L. Mathematical programming in neural networks. Computer Sciences Department Technical Report 1129, University of Wisconsin, 1992.

[16] J. A. Leonard, M. A. Kramer, and L. H. Ungar. Using radial basis functions to approximate a function and its error bounds. IEEE transactions on Neural Networks, 3(4):624-627, 1992.

[17] Yamasaki M. and A. Sakurai. On the capacity of $n-h-1$ networks with sigmoidal functions. In I. Aleksander and J. Taylor, editors, Artificial Neural Networks, 2, volume 1, pages 229-232. Elsevier Science Publishing Company B. V., Amsterdam, 1992.

[18] M. Macintyre and E.D. Sontag. Finiteness results for sigmoidal 'neural' networks. In Proc. 25th Annual Symp. Theory Computing, pages 325-334, San Diego, 1993. 
[19] N. Megiddo. On the complexity of polyhedral separability. Discrete and C'omputational Geometry, 3:325-337, 1988.

[20] M. Mézard and J.-P. Nadal. Learning in feedforward layered networks: The tiling algorithm. Journal of Physics A, 22:2191-2204, 1989.

[21] Bennett K. P. and O. L. Mangasarian. Bilinear separation of two sets in nspace. Computer Sciences Department Technical Report 1109, University of Wisconsin, 1992.

[22] Koiran P. Function approximation using a partition of the input space. In Proceedings of IJCNN'92, pages 883-887, Baltimore, 1992.

[23] T. Poggio and F. Girosi. Networks for approximation and learning. Proceedings of the IEEE, $78(9): 1481-1497,1990$.

[24] D.E. Rumelhart, G.E. Hinton, and R.J. Williams. Learning internal representations by error propagation. In D.E. Rumelhart and J.L. McClelland, editors, Parallel Distributed Processing, volume 1, chapter 8, pages 318-362. MIT Press, Cambridge, 1986. Reprinted in [1].

[25] W. Schiffmann, M. Joost, and R. Werner. Optimierung des backpropagation algorithmus zum training von multilayer perceptrons. Technical Report Fachbericht Physik, 15, Universität Koblenz, 1992. Also available in English.

[26] T. J. Shepard and D. S. Broomhead. Nonlinear signal processing using radial basis functions. Proceedings of the SPIE, 1348:51-61, 1990.

[27] E.D. Sontag. Feedforward nets for interpolation and classification. J. Comp. Syst. Sci., 45:20-48, 1992.

[28] G. Tesauro. Neurogammon wins computer olympiad. Neural Computation, $1: 321-323.1990$.

[29] G. Tesauro. Td-gammon, a self-teaching backgammon program, achieves master-level play. Neural Computation, to appear. 
[30] Maass W. Bounds for the computational power and learning complexity of analog neural nets. In Proc. 25th Annual Symp. Theory Computing, pages 335-344. San Diego, 1993. 

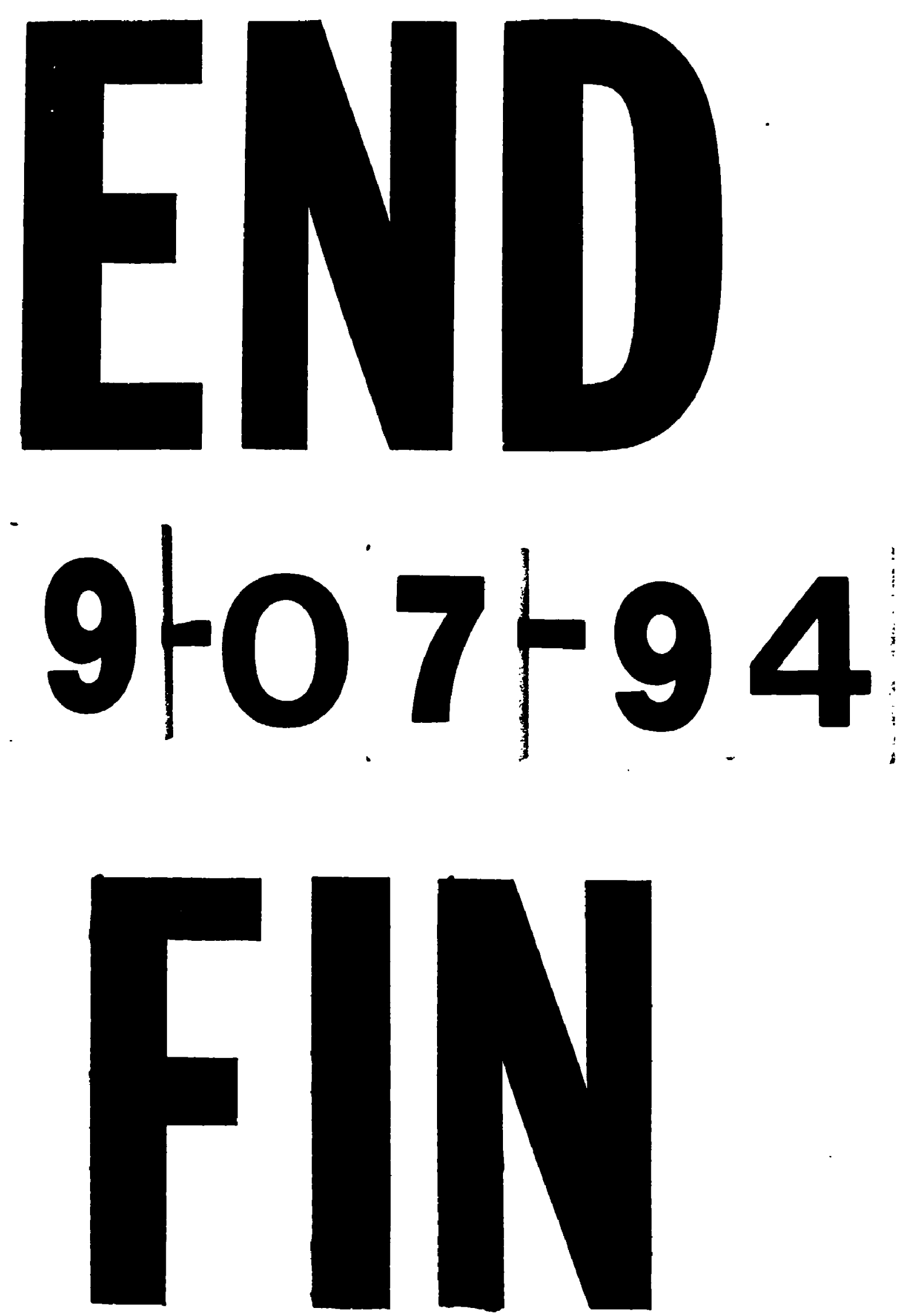